\title{
Dipyrene-Fused Dicyclopenta[a,Inaphthalenes
}

Jason Melidonie,$^{\dagger}$ Evgenia Dmitrieva, ${ }^{\S}$ Ke Zhang, ${ }^{\perp}$ Yubin Fu ${ }^{\dagger}$ Alexey A. Popov,$\S$ Wojciech Pisula, ${ }^{\perp, \pm}$ Reinhard Berger, ${ }^{\dagger}$ Junzhi Liu* ${ }^{*}{ }^{\#}$ and Xinliang Feng ${ }^{*} \dagger$

${ }^{\dagger}$ Center for Advancing Electronics Dresden (cfaed) and Faculty of Chemistry and Food Chemistry and $\star$ Chair of Inorganic Molecular Chemistry, Technische Universität Dresden, 01062 Dresden, Germany.

$\S$ Center of Spectroelectrochemistry, Nanoscale Chemistry, Leibniz Institute for Solid State and Materials Research (IFW Dresden), Helmholtzstraße 20, 01069 Dresden, Germany.

${ }^{\perp}$ Max Planck Institute for Polymer Research, Ackermannweg 10, 55128 Mainz, Germany ${ }^{ \pm}$Lodz University of Technology, Department of Molecular Physics, Faculty of Chemistry, Zeromskiego 116, 90-924, Lodz, Poland.

\# Department of Chemistry and State Key Laboratory of Synthetic Chemistry, The University of Hong Kong, Pokfulam Road, Hong Kong, China

*Corresponding authors. E-mail:

xinliang.feng@tu-dresden.de, and juliu@hku.hk 


\section{Table of Content}

1) HR-MALDI-TOF mass spectrometry $\quad$ S3

2) NMR spectroscopy

3) Experimental Values of Optoelectronic Data $\quad$ S21

$\begin{array}{lr}\text { 4) EPR spectra } & \text { S22 }\end{array}$

$\begin{array}{ll}\text { 5) Spectroelectrochemistry } & \text { S23 }\end{array}$

$\begin{array}{lr}\text { 6) Computation } & \text { S24 }\end{array}$

$\begin{array}{ll}\text { 6.1 ACID plots } & \text { S25 }\end{array}$

$\begin{array}{ll}\text { 6.2 Biradical character } & \text { S27 }\end{array}$

6.3 Visualization of the single occupied molecular orbitals $\quad$ S28

6.4 Cartesian coordinates of all optimized structures $\quad$ S30

$\begin{array}{ll}\text { 6.5 Spin density } & \text { S60 }\end{array}$

$\begin{array}{lr}\text { 7) References } & \text { S70 }\end{array}$ 


\section{1) HR-MALDI-TOF mass spectrometry}
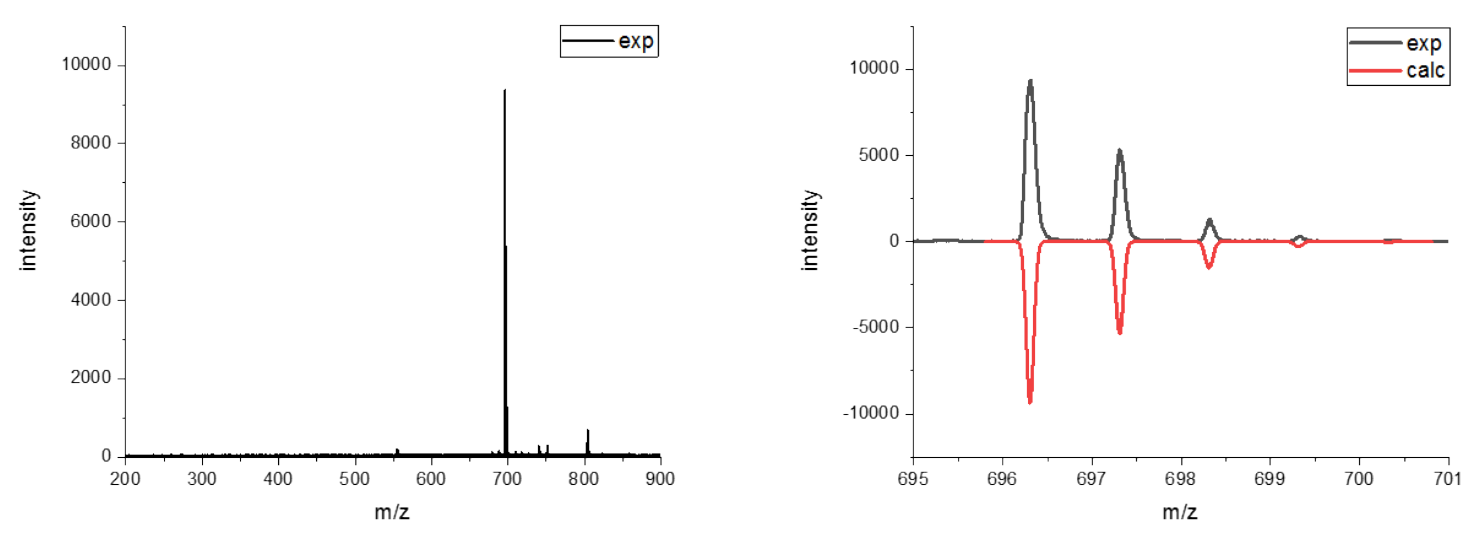

Figure S1. a) HR-MALDI-TOF spectrum of 5a. b) HR-MALDI-TOF measurement of 5a (black line) is in agreement to the expected isotopic distribution pattern (red line).
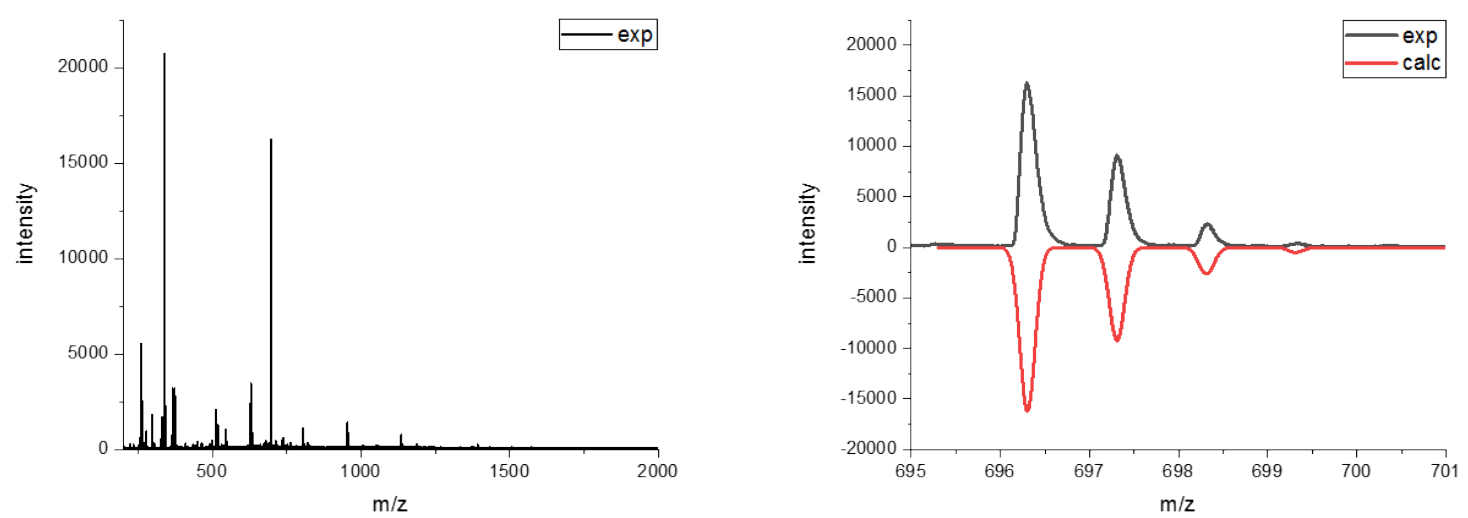

Figure S2. a) HR-MALDI-TOF spectrum of 5b. b) HR-MALDI-TOF measurement of $\mathbf{5 b}$ (black line) is in agreement to the expected isotopic distribution pattern (red line). 

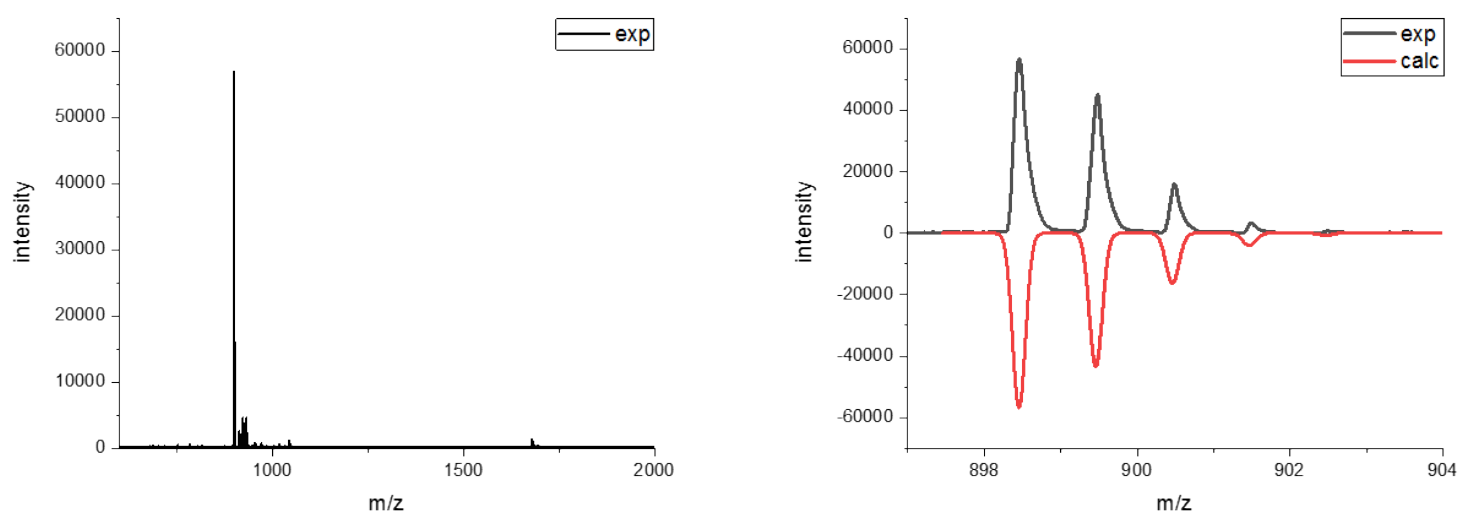

Figure S3. a) HR-MALDI-TOF spectrum of 1a. b) HR-MALDI-TOF measurement of 1a (black line) is in agreement to the expected isotopic distribution pattern (red line).
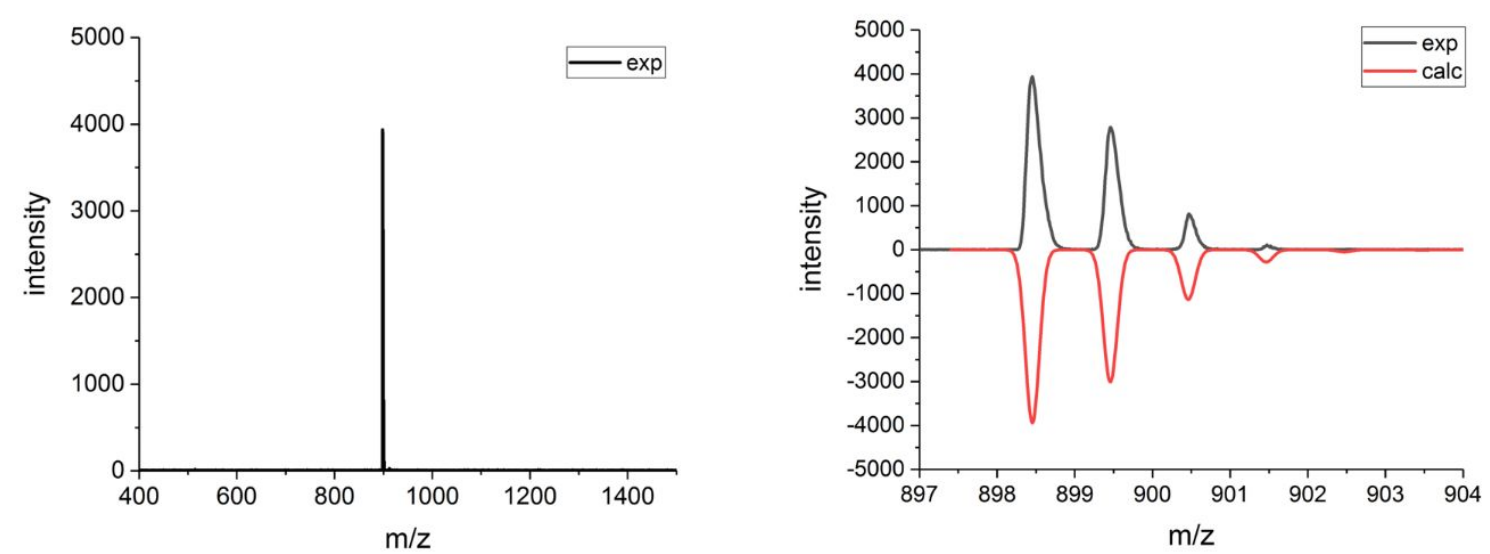

Figure S4. a) HR-MALDI-TOF spectrum of 1b. b) HR-MALDI-TOF measurement of 1b (black line) is in agreement to the expected isotopic distribution pattern (red line). 


\section{2) NMR spectroscopy}

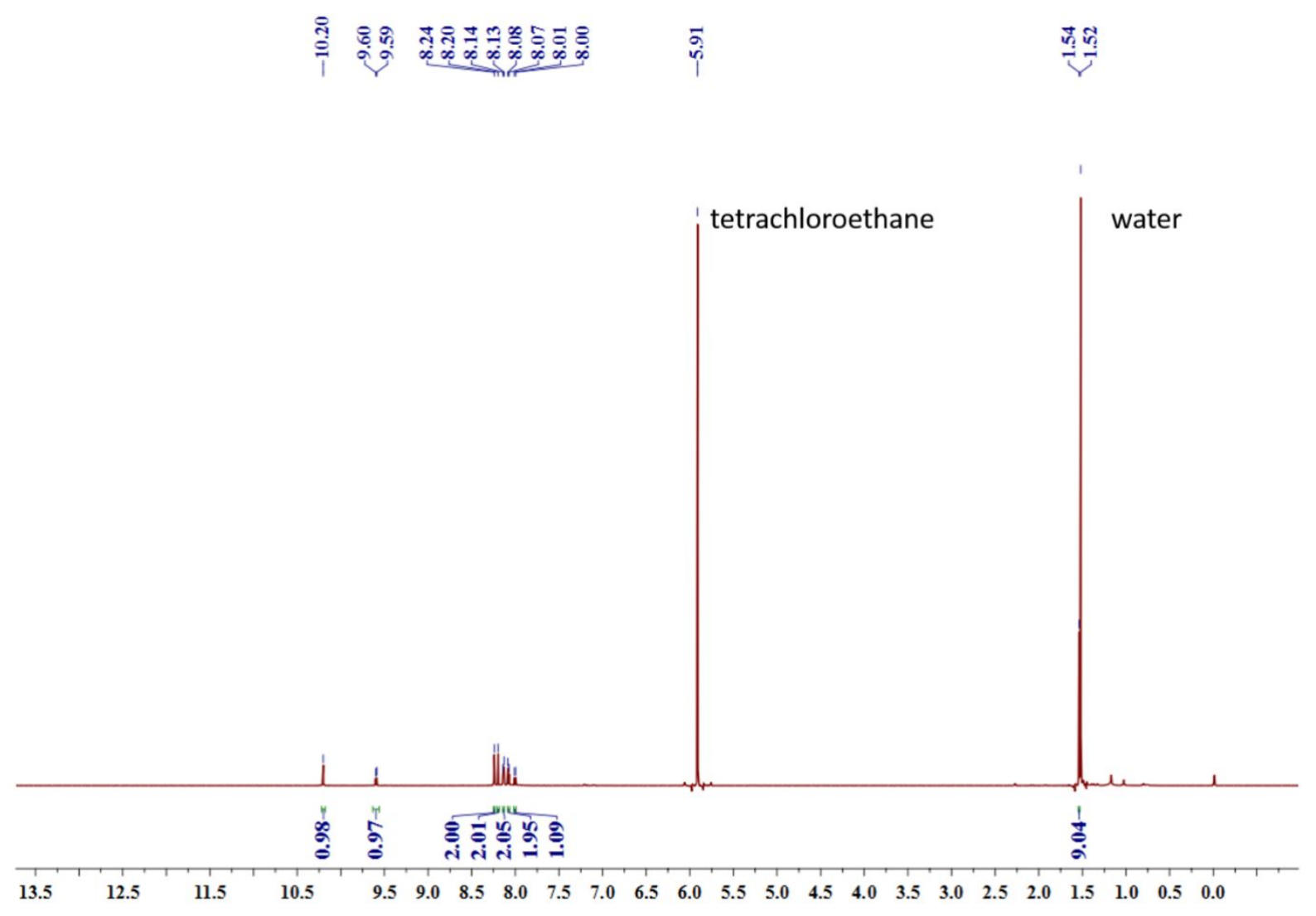

Figure S5. ${ }^{1} \mathrm{H}-\mathrm{NMR}$ spectrum of 5a dissolved in tetrachloroethane-d2, $600 \mathrm{MHz}$, room temperature. 


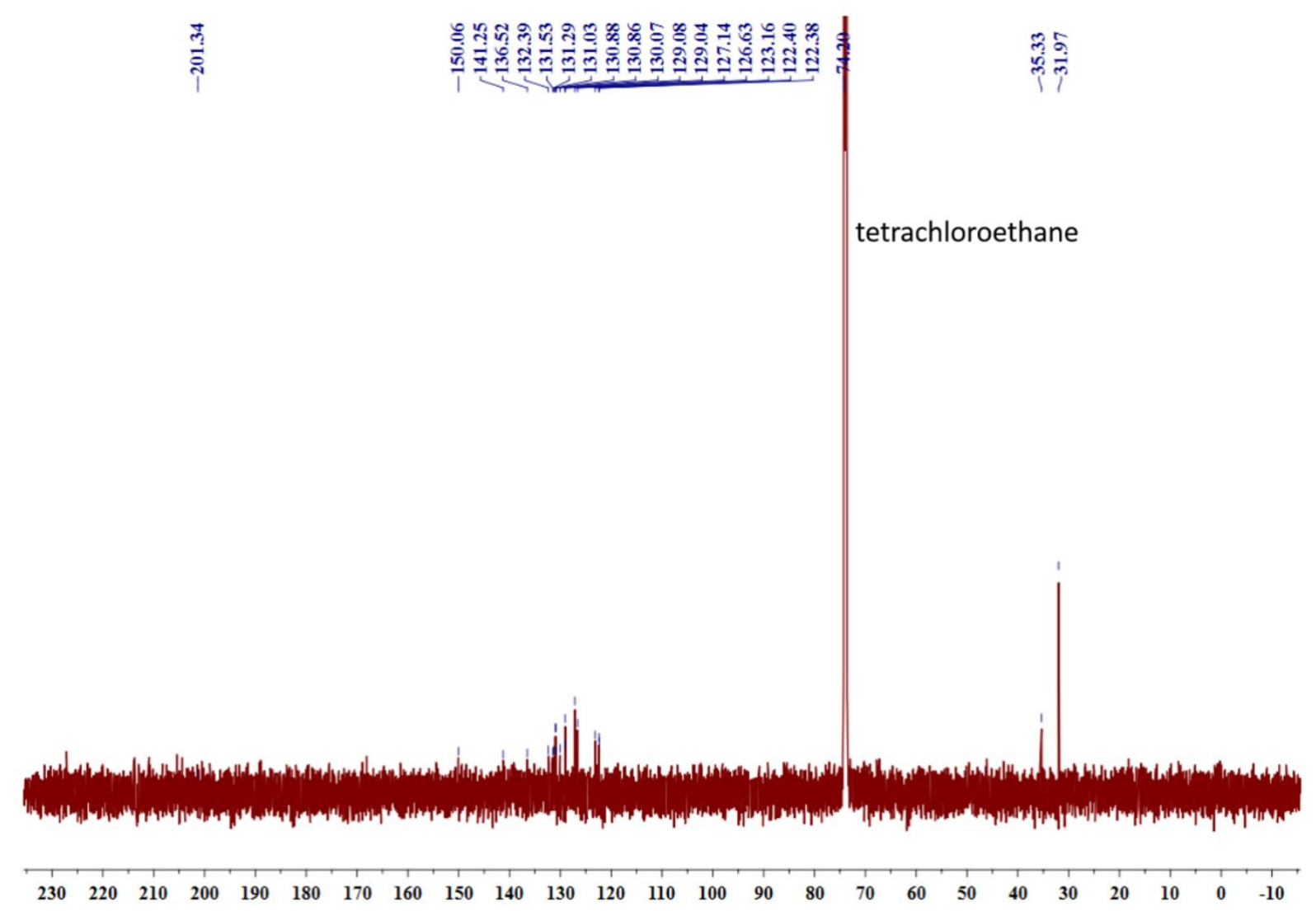

Figure S6. ${ }^{13} \mathrm{C}-\mathrm{NMR}$ spectrum of 5a dissolved in tetrachloroethane-d2, $151 \mathrm{MHz}$, room temperature. 


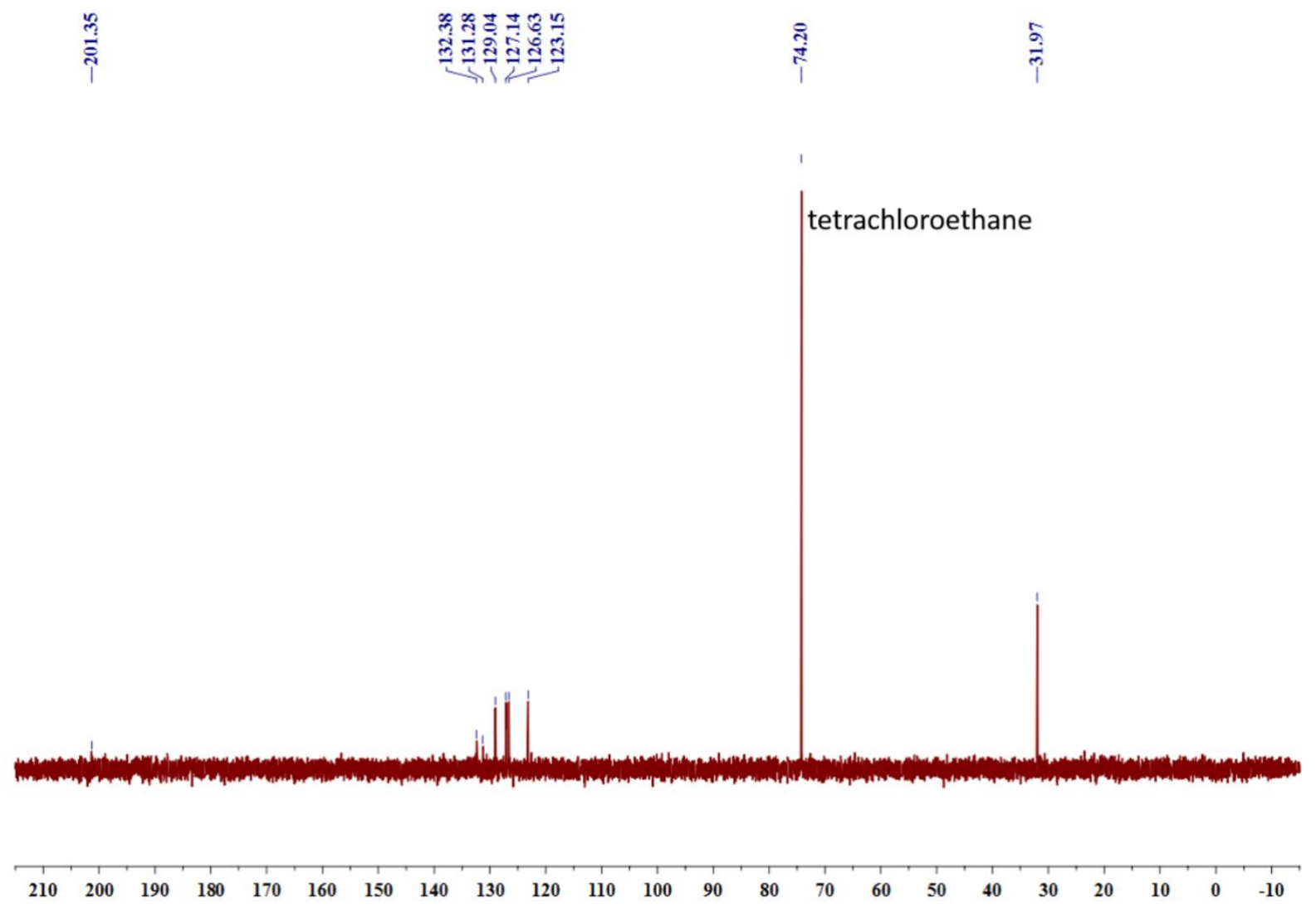

Figure S7. ${ }^{13} \mathrm{C}$-DEPT-NMR spectrum of 5a dissolved in tetrachloroethane-d2, $151 \mathrm{MHz}$, room temperature. 


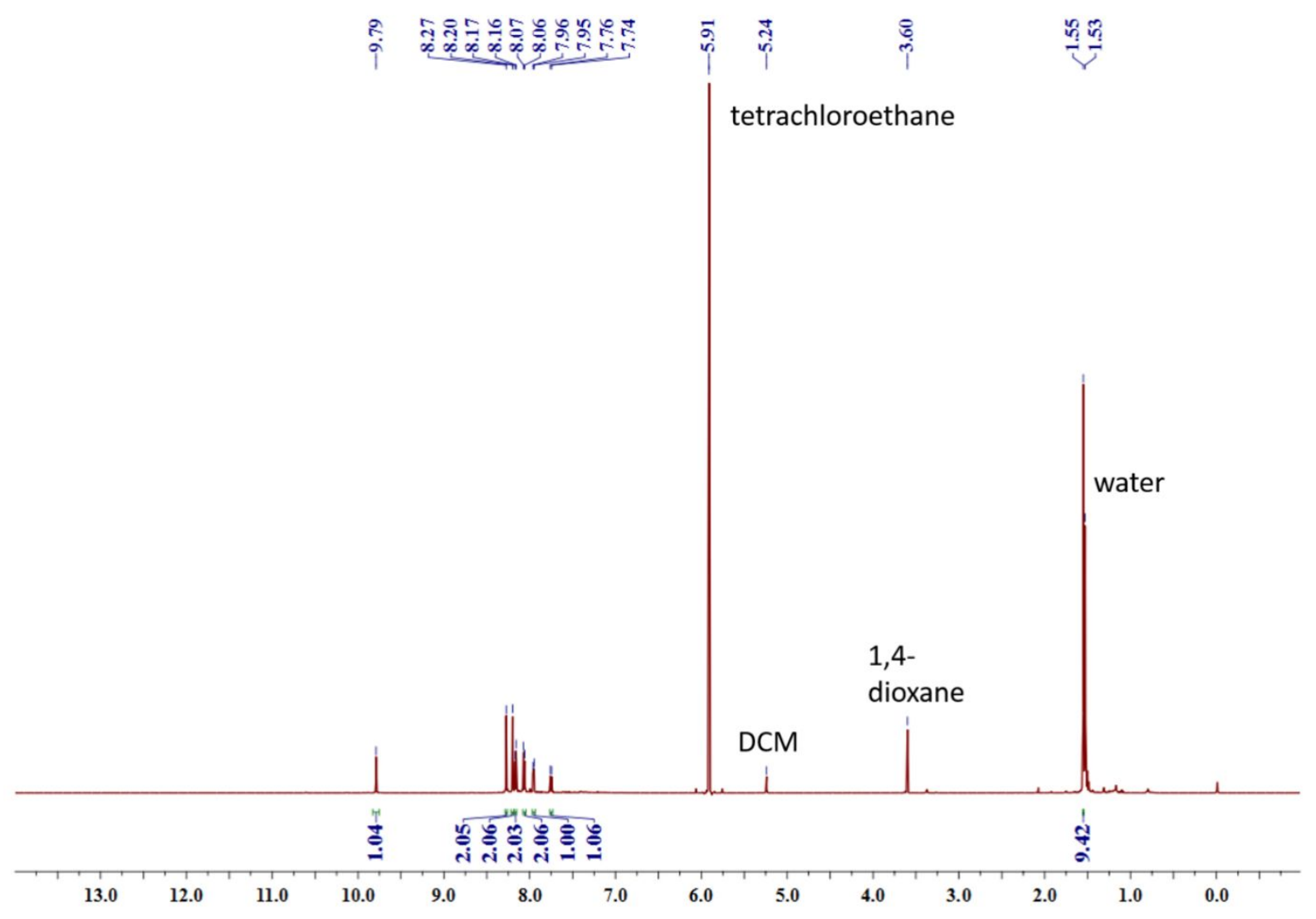

Figure S8. ${ }^{1} \mathrm{H}-\mathrm{NMR}$ spectrum of $\mathbf{5 b}$ dissolved in tetrachloroethane-d2, $600 \mathrm{MHz}$, room temperature. 


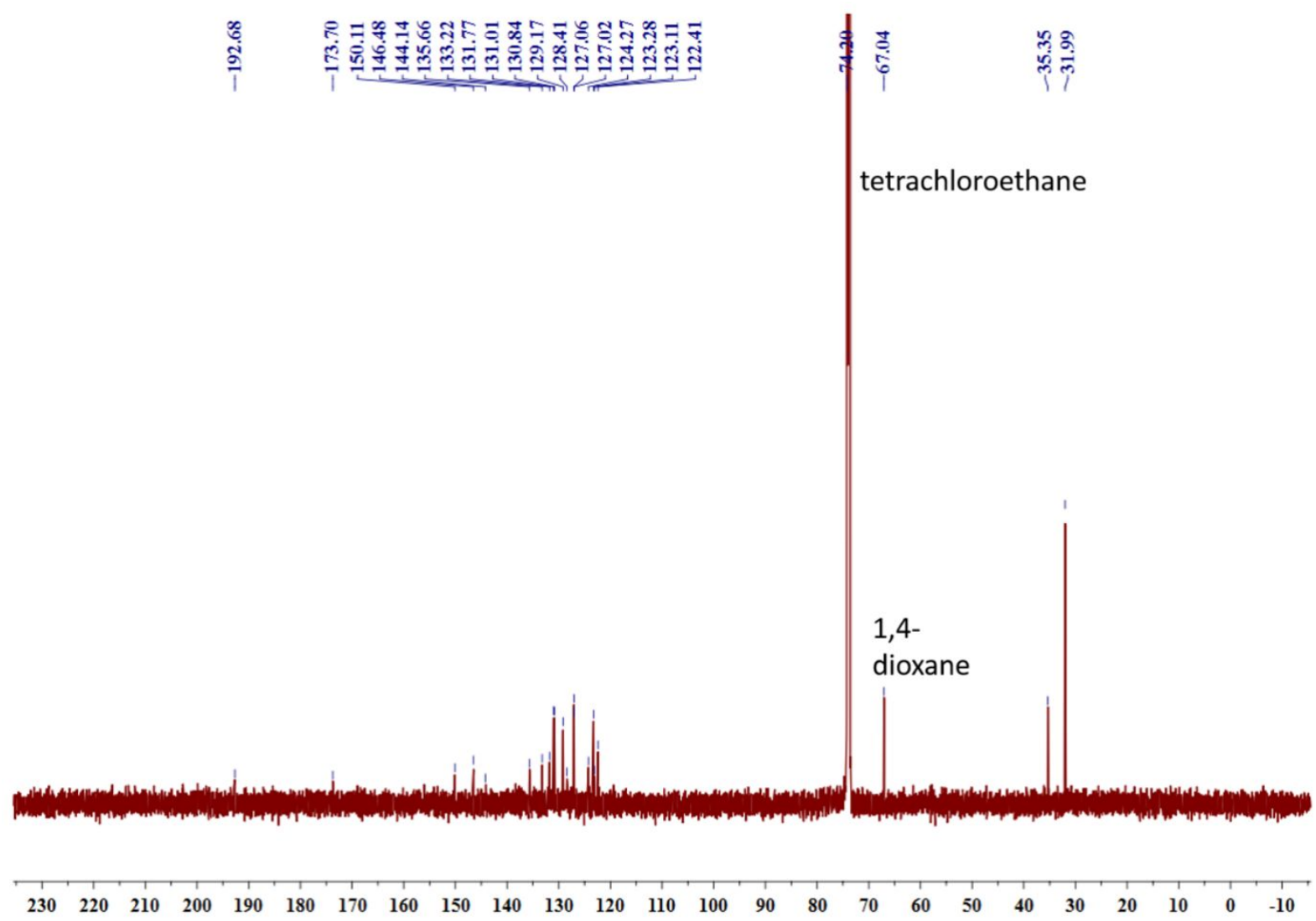

Figure S9. ${ }^{13} \mathrm{C}-\mathrm{NMR}$ spectrum of $\mathbf{5 b}$ dissolved in tetrachloroethane-d2, $151 \mathrm{MHz}$, room temperature. 


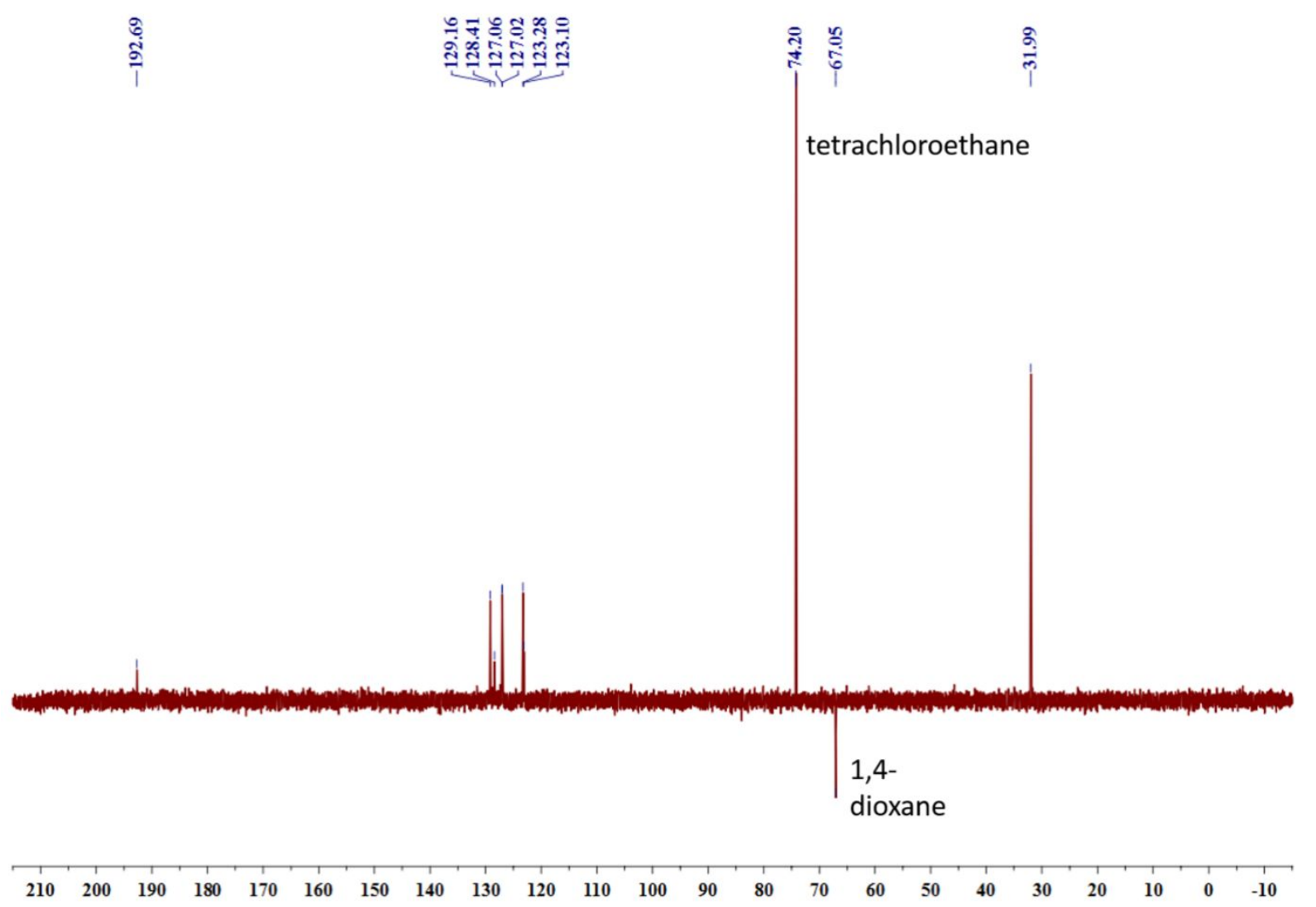

Figure S10. ${ }^{13} \mathrm{C}-\mathrm{DEPT} 135-\mathrm{NMR}$ spectrum of $\mathbf{5 b}$ dissolved in tetrachloroethane-d2, 151 $\mathrm{MHz}$, room temperature. 


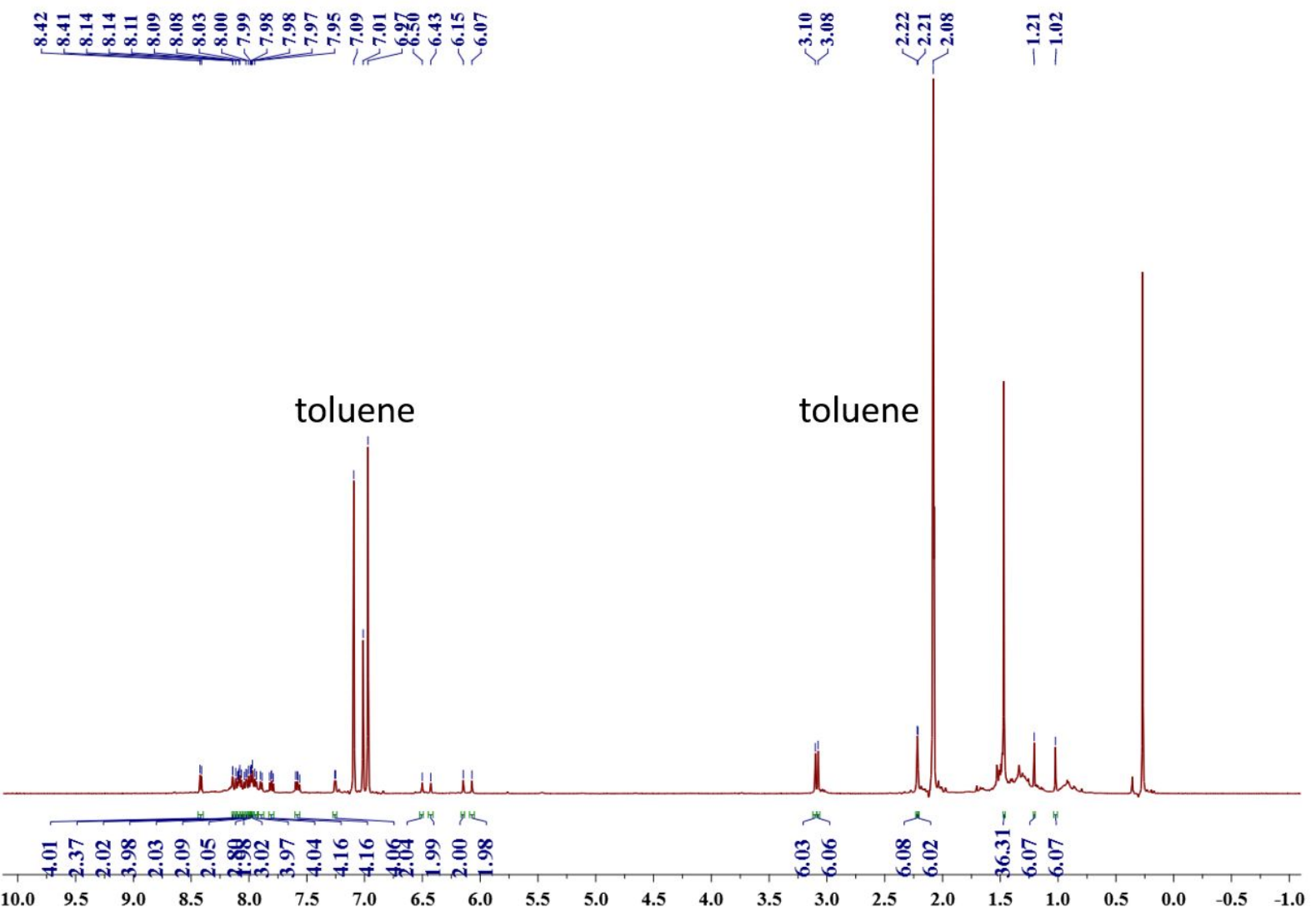

Figure S11. ${ }^{1} \mathrm{H}-\mathrm{NMR}$ spectrum of 6a dissolved in toluene-d8, $600 \mathrm{MHz}$, room temperature.

The compound appears as an isomeric 1:1 mixture. 
toluene

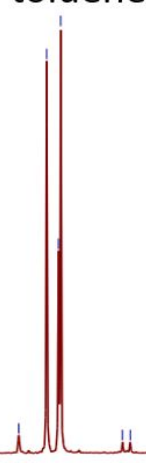

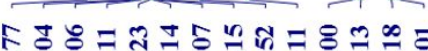

$\prod_{4=1}$

Figure S12. ${ }^{1} \mathrm{H}-\mathrm{NMR}$ spectrum of $\mathbf{6 a}$ dissolved in toluene-d8, $151 \mathrm{MHz}, 353.15 \mathrm{~K}$. The compound still appears as an isomeric 1:1 mixture at higher temperatures. The HT measurement of $6 \mathbf{a}$ can be regarded as a stability test for the compound at $80{ }^{\circ} \mathrm{C}$. 
Since compound $\mathbf{6 a}$ is stable at $80^{\circ} \mathrm{C}$, an in situ high temperature NMR measurement of $\mathbf{6 a}$ in the presence of two equivalents DDQ was carried out in a NMR tube. The tube was gradually heated to $80{ }^{\circ} \mathrm{C}$ in a $600 \mathrm{MHz}$ NMR spectrometer. As soon as the DDQ was added the first measurement was applied at room temperature. It was shown that the signals of $6 \mathbf{6}$ already started to vanish which is a hint for the evolution of 1a. Since 1a is expected to be a biradical the NMR spectra should become silent. By subsequent heating to $80{ }^{\circ} \mathrm{C}$ all the signals of $6 \mathbf{6}$ disappeared. The temperature was kept at $80{ }^{\circ} \mathrm{C}$ for one hour. After cooling down to room temperature, another spectra was recorded proving that 1a still exists due to the silent spectrogram.

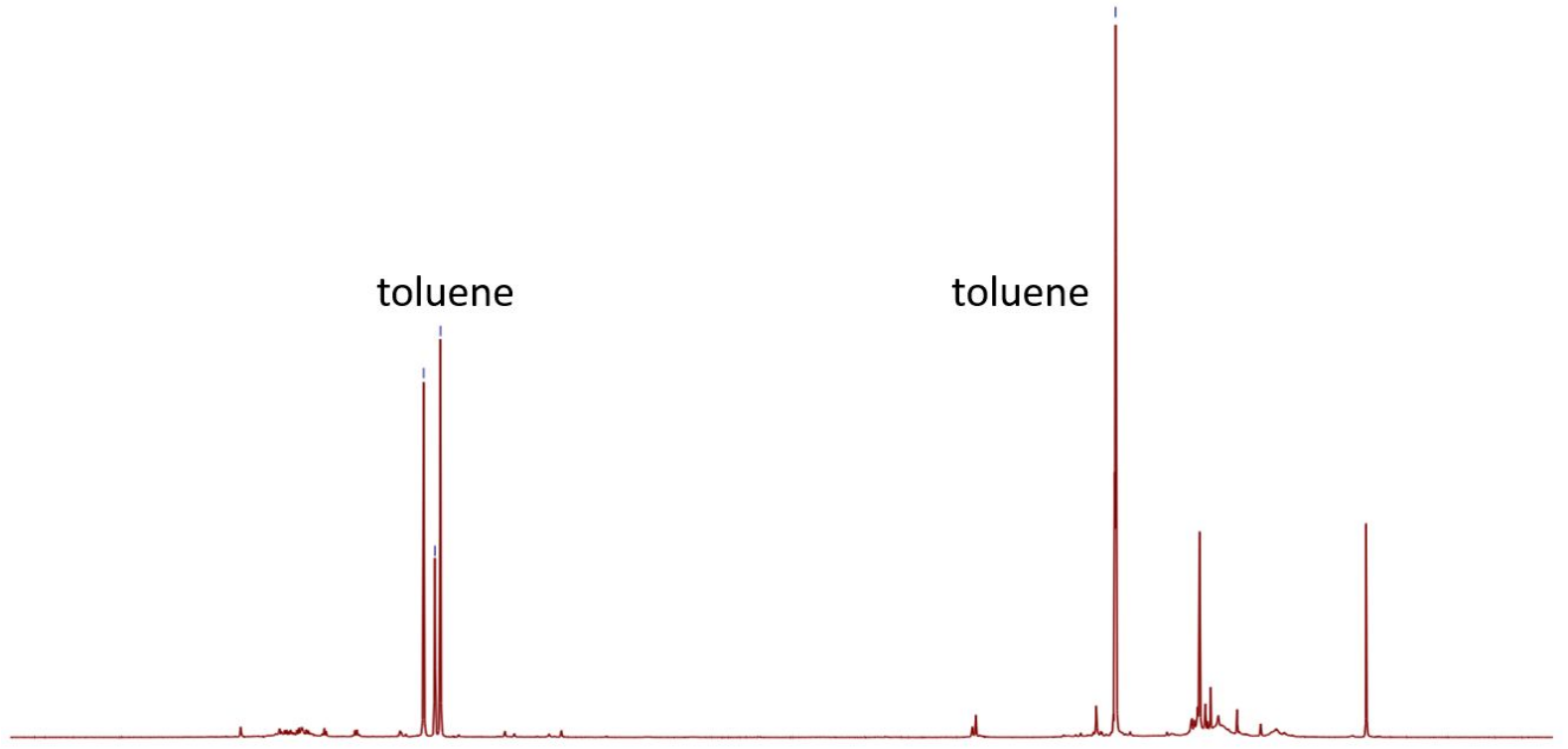

$\begin{array}{lllllllllllllllllllllll}10.0 & 9.5 & 9.0 & 8.5 & 8.0 & 7.5 & 7.0 & 6.5 & 6.0 & 5.5 & 5.0 & 4.5 & 4.0 & 3.5 & 3.0 & 2.5 & 2.0 & 1.5 & 1.0 & 0.5 & 0.0 & -0.5 & -1.0\end{array}$ Figure S13. ${ }^{1} \mathrm{H}-\mathrm{NMR}$ spectrum of $\mathbf{6 a}$ dissolved in toluene-d8 in the presence of 2 eq DDQ, $151 \mathrm{MHz}$, room temperature. 


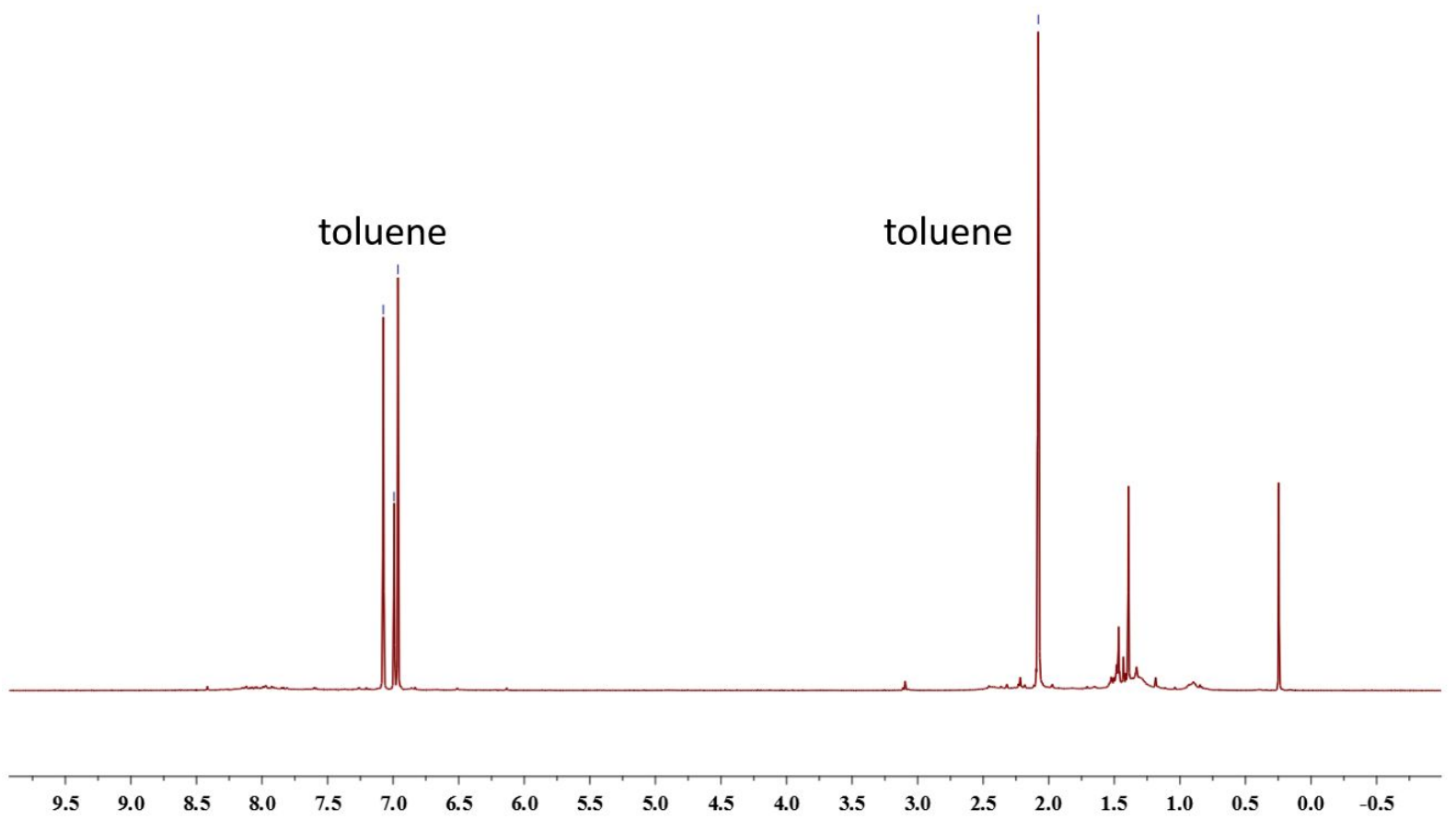

Figure S14. ${ }^{1} \mathrm{H}-\mathrm{NMR}$ spectrum of $\mathbf{6 a}$ dissolved in toluene-d8 in the presence of 2 eq DDQ, $151 \mathrm{MHz}, 319 \mathrm{~K}$. 


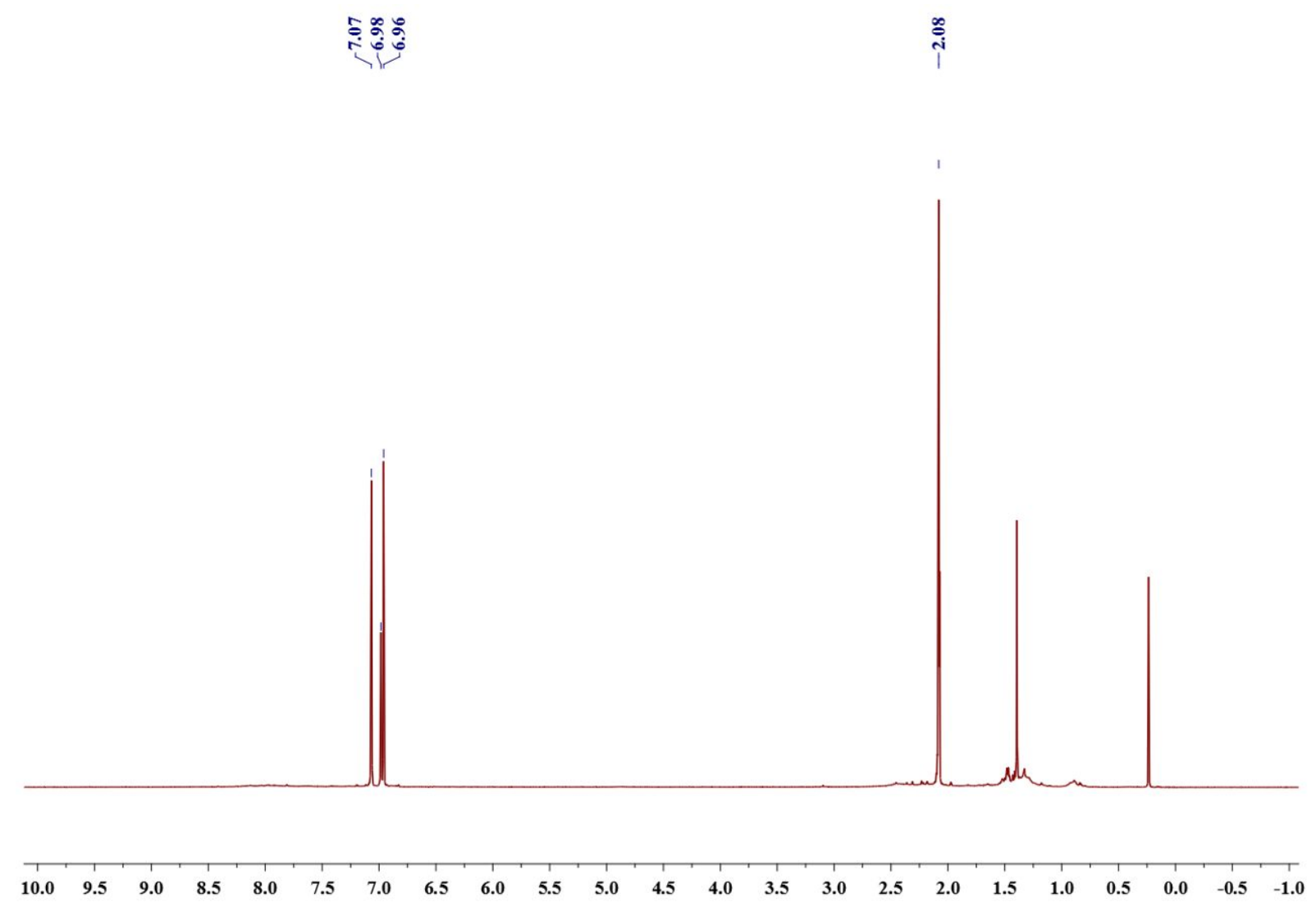

Figure S15. ${ }^{1} \mathrm{H}-\mathrm{NMR}$ spectrum of $\mathbf{6 a}$ dissolved in toluene-d8 in the presence of 2 eq DDQ, $151 \mathrm{MHz}, 333 \mathrm{~K}$. 
toluene
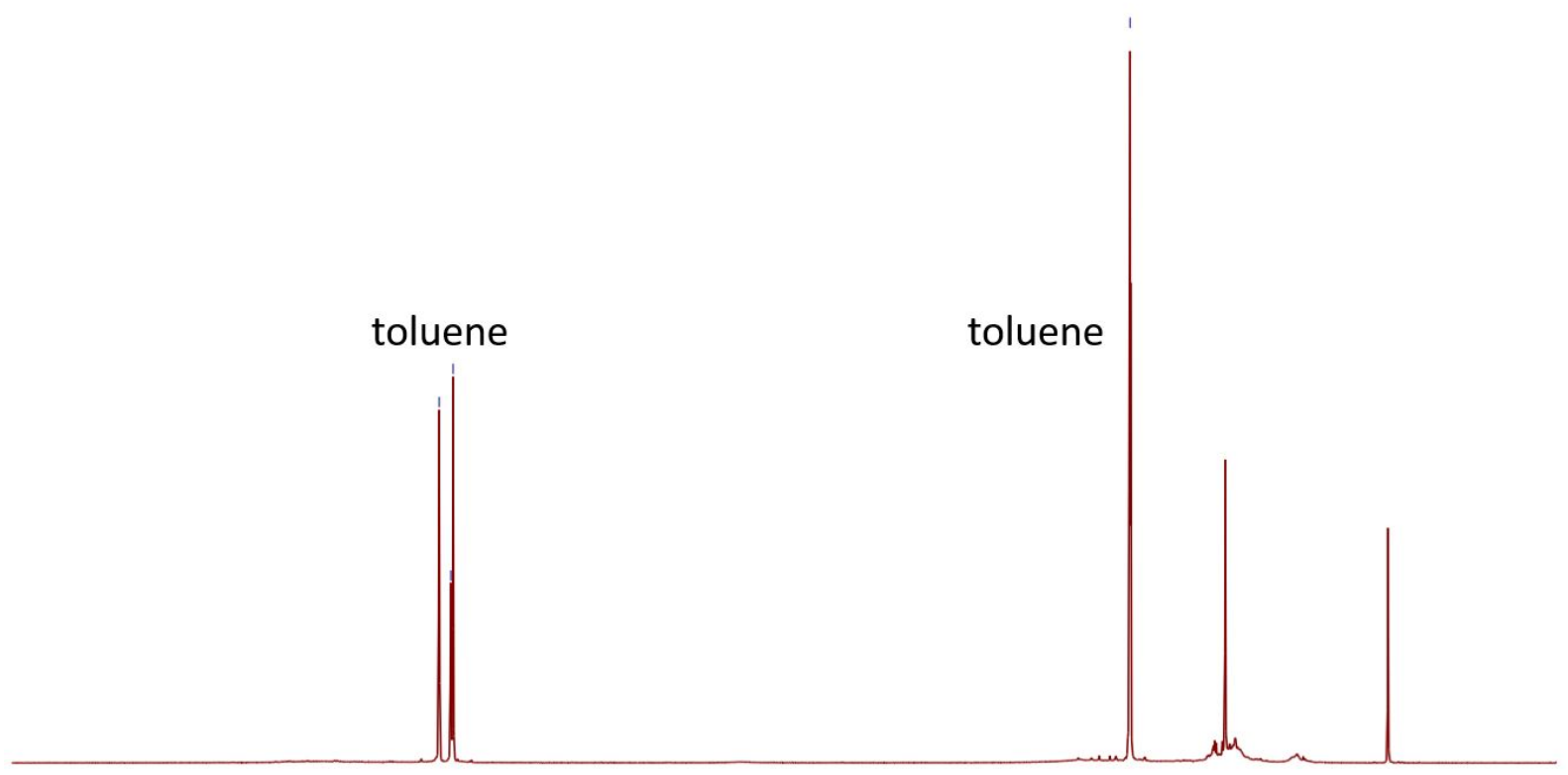

$\begin{array}{llllllllllllllllllllll}10.0 & 9.5 & 9.0 & 8.5 & 8.0 & 7.5 & 7.0 & 6.5 & 6.0 & 5.5 & 5.0 & 4.5 & 4.0 & 3.5 & 3.0 & 2.5 & 2.0 & 1.5 & 1.0 & 0.5 & 0.0 & -0.5\end{array}$

Figure S16. ${ }^{1} \mathrm{H}-\mathrm{NMR}$ spectrum of $\mathbf{6 a}$ dissolved in toluene-d8 in the presence of 2 eq DDQ, $151 \mathrm{MHz}, 353 \mathrm{~K}$. 

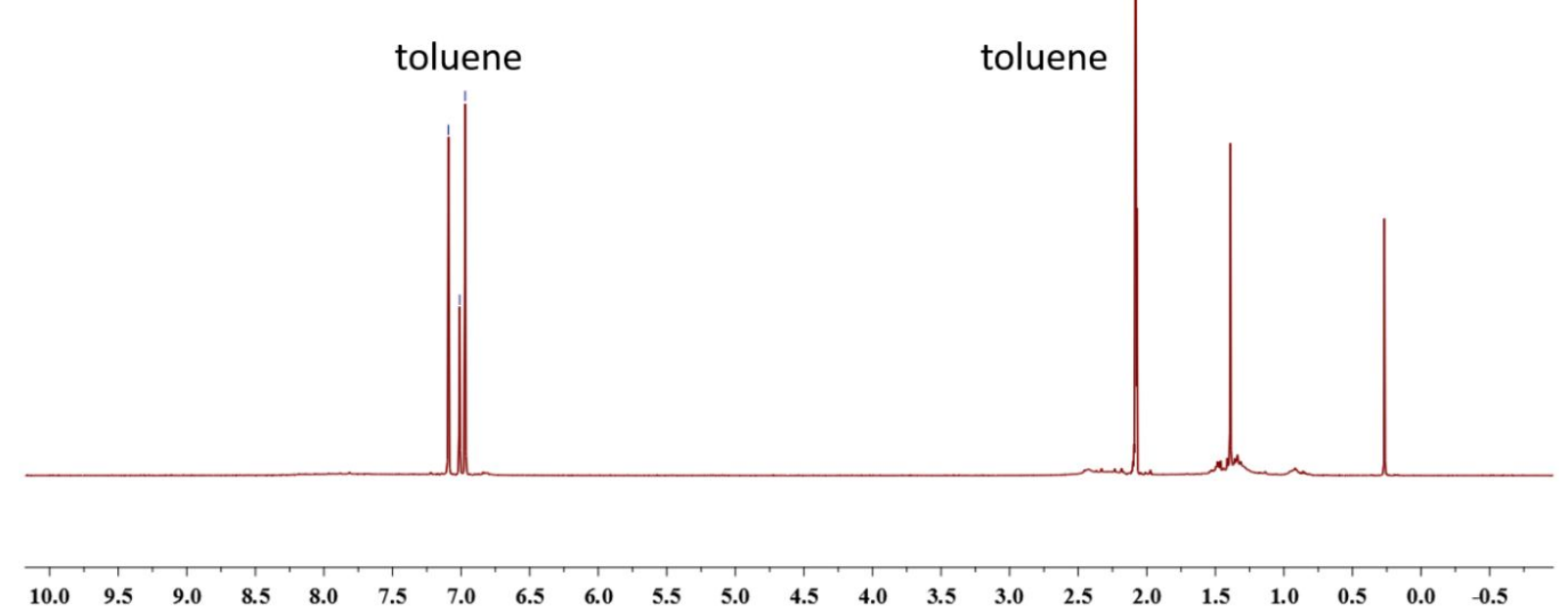

Figure S17. ${ }^{1} \mathrm{H}-\mathrm{NMR}$ spectrum of $\mathbf{6 a}$ dissolved in toluene-d8 in the presence of 2 eq DDQ, $151 \mathrm{MHz}$, after cooling down to $296 \mathrm{~K}$. 


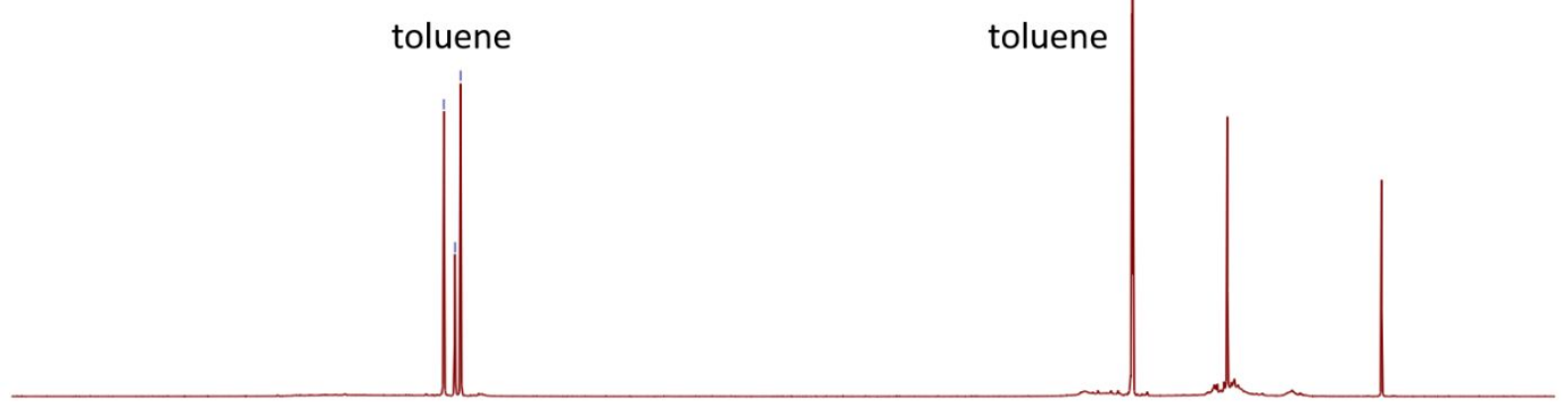

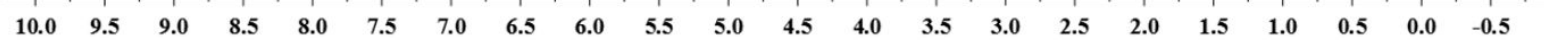

Figure S18. ${ }^{1} \mathrm{H}-\mathrm{NMR}$ spectrum of $6 \mathrm{a}$ dissolved in toluene-d8 in the presence of 2 eq DDQ, $151 \mathrm{MHz}$, after cooling down to $253 \mathrm{~K}$. 


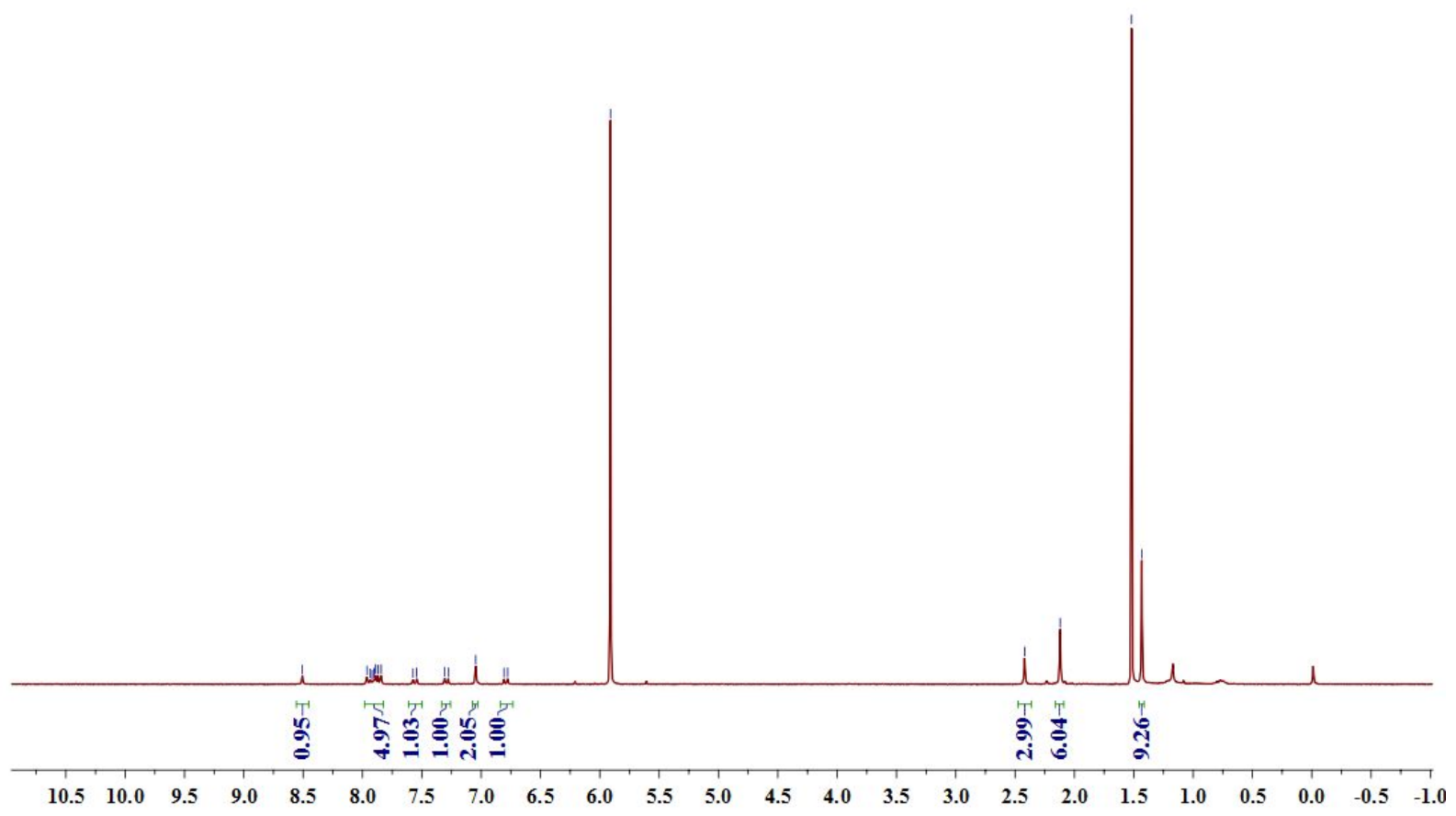

Figure S19. ${ }^{1} \mathrm{H}-\mathrm{NMR}$ spectrum of $\mathbf{1 b}$ dissolved in tetrachloroethane-d2, $600 \mathrm{MHz}$, room temperature. 


\section{b) $373 \mathrm{~K}$}

\section{c) $353 \mathrm{~K}$}

d) $333 \mathrm{~K}$
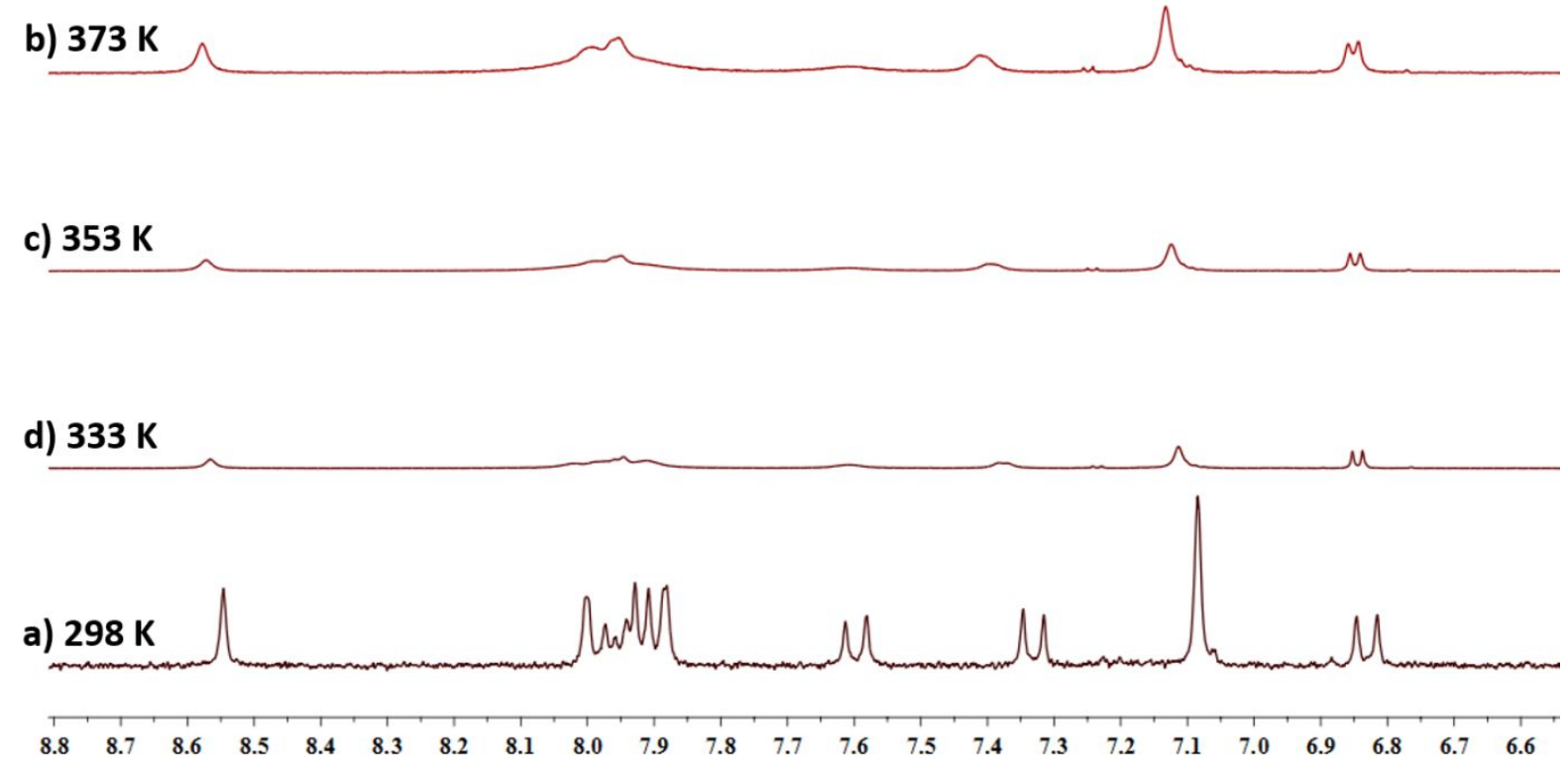

Figure S20. ${ }^{1} \mathrm{H}-\mathrm{NMR}$ spectrum of $\mathbf{1 b}$ dissolved in tetrachloroethane-d2, a) at room temperature, b) at $373 \mathrm{~K}, \mathrm{c}$ ) at $353 \mathrm{~K}, \mathrm{~d}$ ) at $333 \mathrm{~K}$. 


\section{3) Experimental Values of Optoelectronic Data}

Table S1. Experimental Values of Optoelectronic Data

\begin{tabular}{|c|c|c|c|c|c|}
\hline \multirow[b]{2}{*}{ compd } & \multicolumn{2}{|c|}{ UV-vis-NIR } & \multicolumn{3}{|l|}{$\mathrm{CV}$} \\
\hline & $\begin{array}{l}\lambda_{\text {onset }^{a}} \\
(\mathrm{~nm})\end{array}$ & $\begin{array}{l}\Delta E_{\mathrm{g}} \mathrm{opt}, b \\
(\mathrm{eV})\end{array}$ & $\begin{array}{l}E_{\mathrm{HOMO}}{ }^{c} \\
(\mathrm{eV})\end{array}$ & $\begin{array}{l}E_{\mathrm{LUMO}}{ }^{d} \\
(\mathrm{eV})\end{array}$ & $\begin{array}{l}\Delta E_{\mathrm{g}}^{\mathrm{el}, e} \\
(\mathrm{eV})\end{array}$ \\
\hline $1 \mathbf{a}$ & 1212 & 1.02 & -4.71 & -3.70 & 1.01 \\
\hline $1 b$ & 1087 & 1.14 & -4.84 & -3.75 & 1.09 \\
\hline
\end{tabular}

${ }^{a}$ Wavelength estimated from onset potential; ${ }^{b}$ optical energy gap calculated from the UV-visNIR absorption maxima using the Planck-Einstein relation; ${ }^{c} \mathrm{HOMO}$ estimated from the onset potential of the first oxidation wave; ${ }^{d}$ LUMO estimated from the onset potential of the first reduction wave; ${ }^{e}$ energy gap calculated according to $\Delta E_{\mathrm{g}}=E_{\mathrm{HOMO}}-E_{\mathrm{LUMO}}$. 


\section{4) EPR spectra}

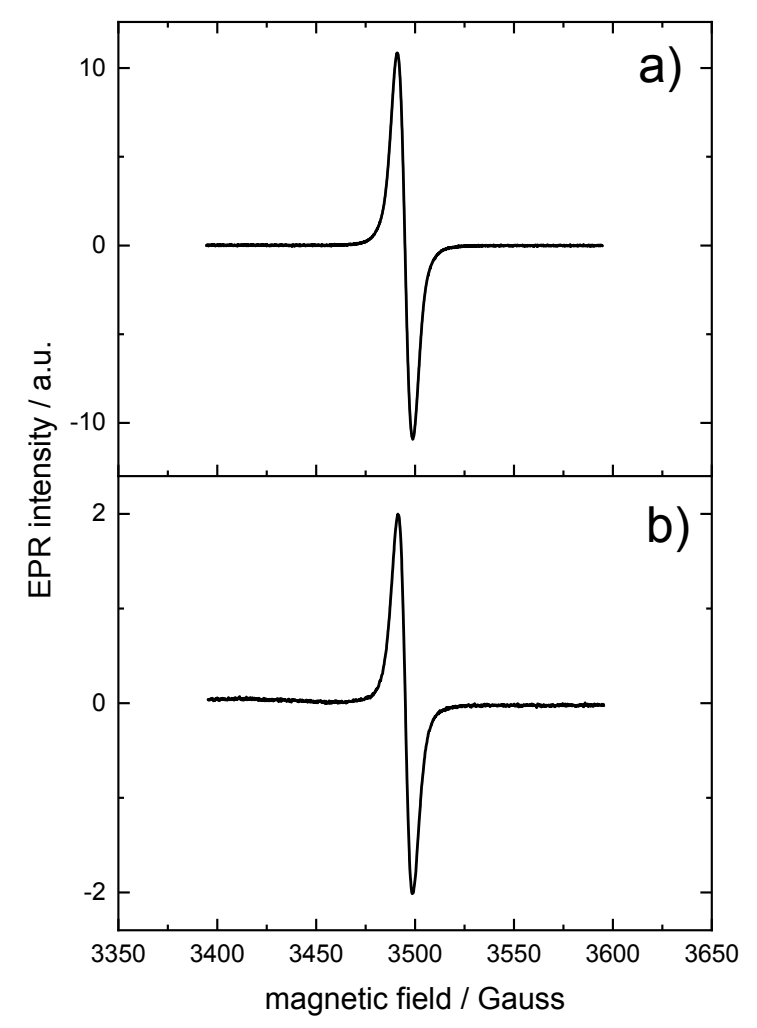

Figure S21. a) EPR spectra of 1a. b) EPR spectra of 1b. 


\section{5) Spectroelectrochemistry}
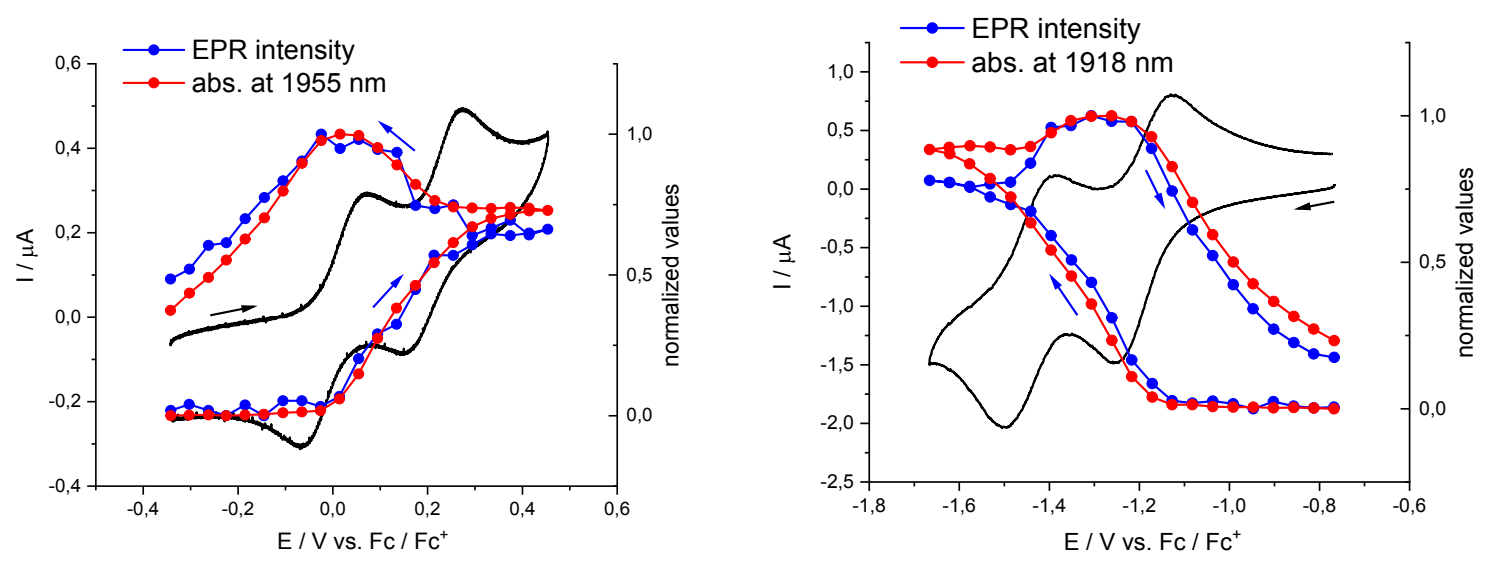

Figure S22. Potential profile of the EPR intensity and absorption bands for 1a.
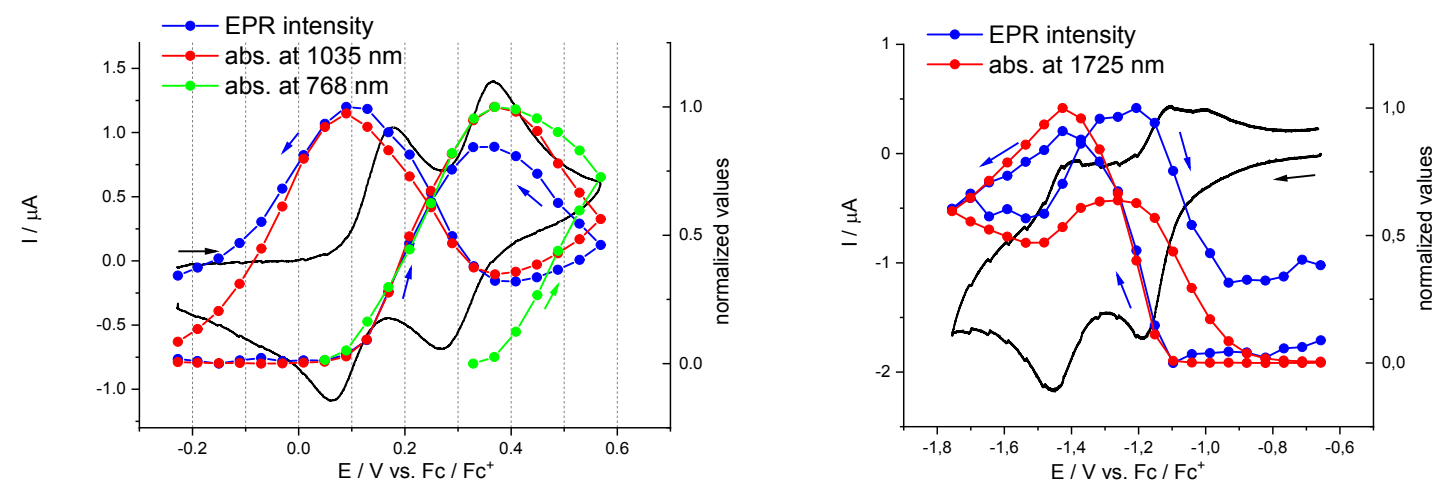

Figure S23. Potential profile of the EPR intensity and absorption bands for $\mathbf{1 b}$. 


\section{6) Computation}

All density functional theory (DFT) calculation was performed using the Gaussian 09 program. ${ }^{[1]}$ The geometry optimization of compound $\mathbf{1 a}$ or compound $\mathbf{1 b}$ in the ground state was optimized by the UB3LYP/6-31G* and B3LYP/6-31G*, respectively. All geometry optimization was done in the gas phase.

Singlet biradical character index (y) was estimated using a symmetry-broken UB3LYP/6$31 \mathrm{G}(\mathrm{d})$ method after geometry optimization. The following equation showed the calculate method:[2]

$$
y=1-2 T\left(1+T^{2}\right)
$$

$\mathrm{T}$ is the orbital overlap between the corresponding orbital pairs (HOMO and LUMO), it can be calculated by using the following equation:

$$
T=\left(n_{H O M O}-n_{L U M O}\right) / 2
$$

Anisotropy of the induced current density (ACID) plots were calculated by Herges's method. ${ }^{[3]}$ Nucleus independent chemical shifts (NICS) values were calculated using the standard gauge invariant atomic orbital (GIAO) ${ }^{[4]}$ method at the level of B3LYP/6$31+\mathrm{G}(2 \mathrm{~d}, \mathrm{p})$ and UB3LYP/6-31+G(2d,p), respectively. All NICS values were averaged by two positions (above and below the plane) of each molecule. 
6.1 ACID plots

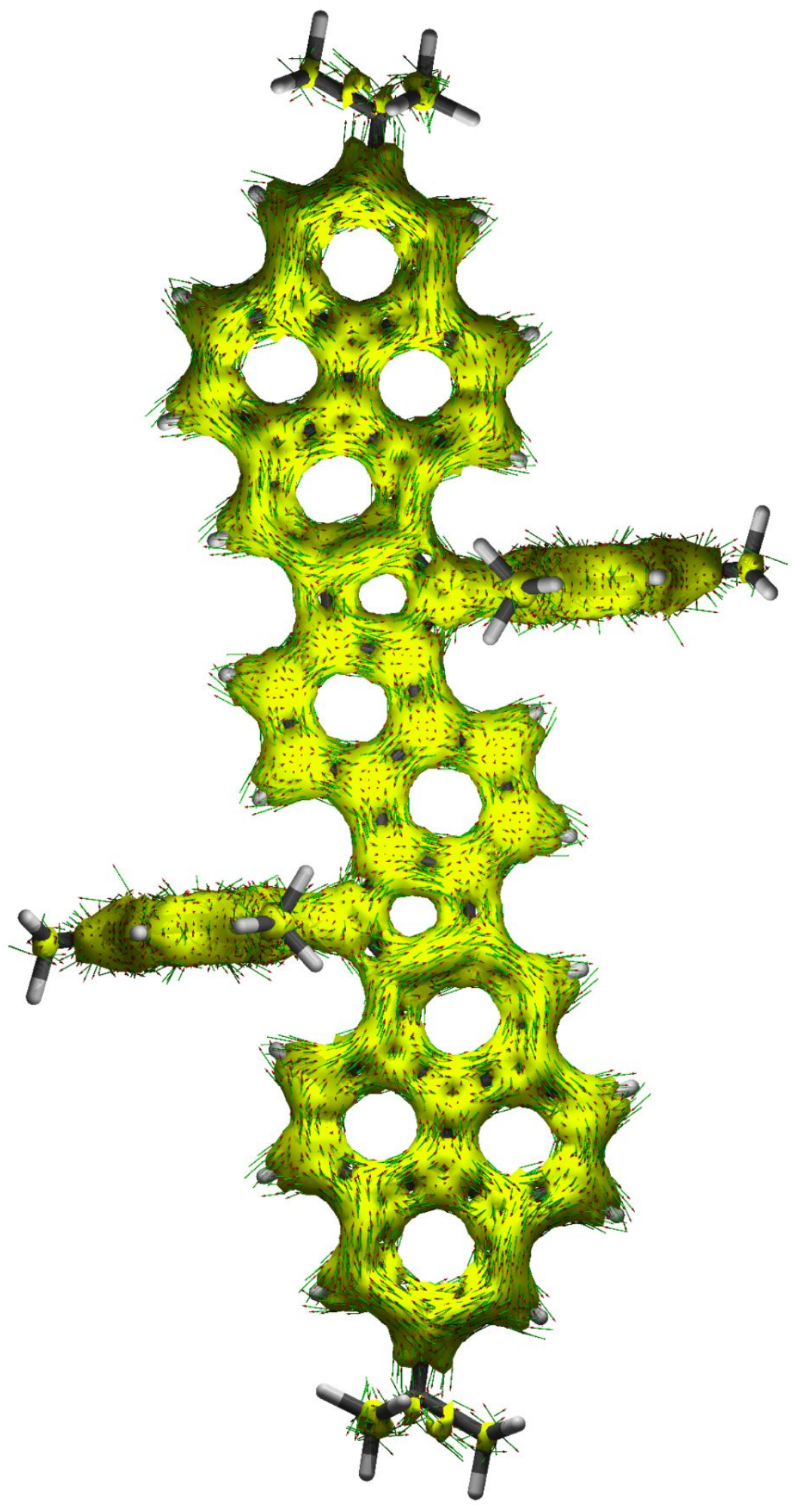

Figure S24. ACID plot of 1a. 


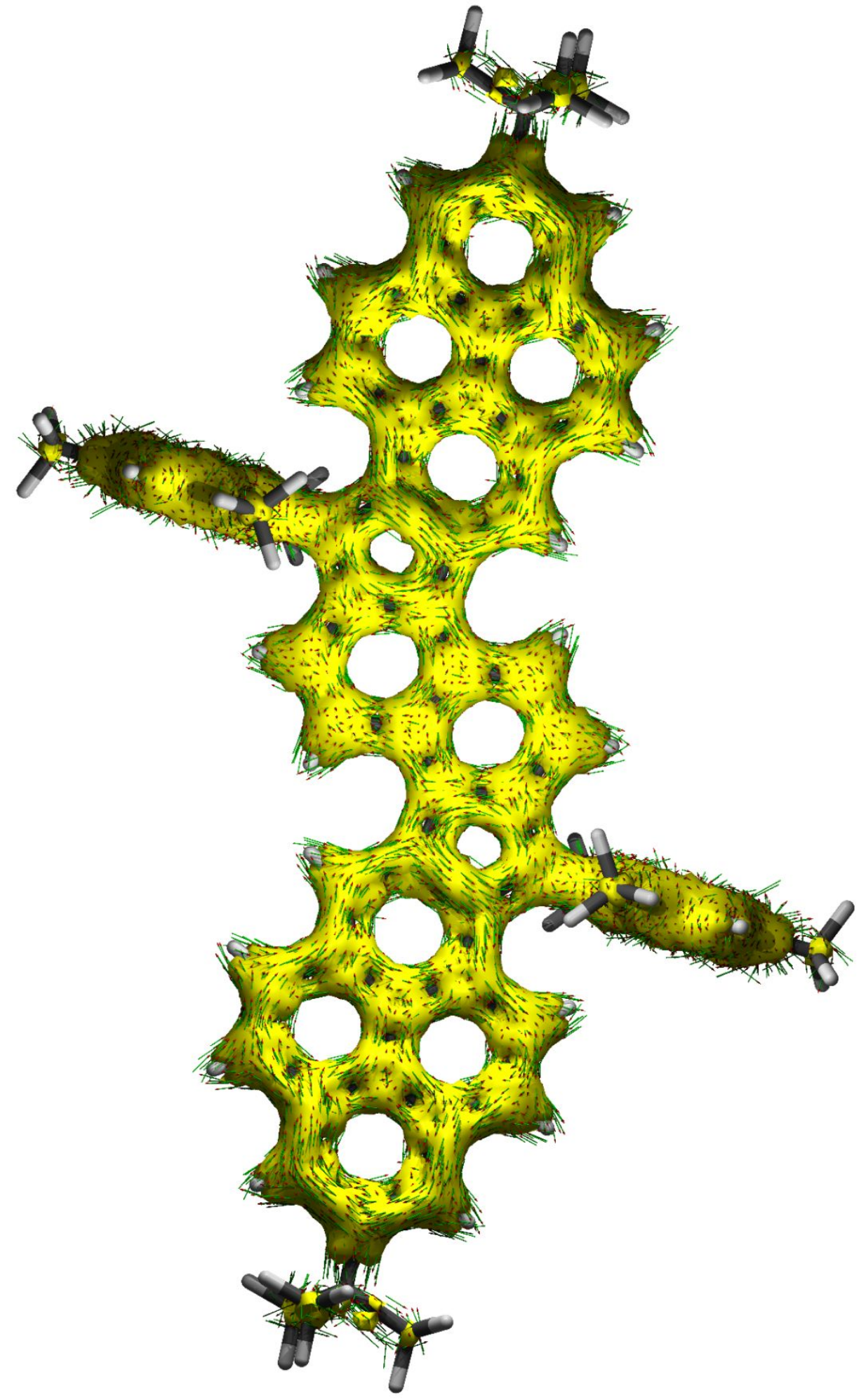

Figure S25. ACID plot of $\mathbf{1 b}$. 


\subsection{Biradical character}

Table S2. Calculation of the biradical character of $1 \mathbf{a}$

\begin{tabular}{|l|l|l|l|l|}
\hline $\begin{array}{l}E(\text { Close shell } \\
\text { state) }\end{array}$ & $\begin{array}{l}E \text { (Open shell } \\
\text { singlet biradical } \\
\text { state) }\end{array}$ & $\begin{array}{l}E \text { (Open shell } \\
\text { triplelet state) }\end{array}$ & $\begin{array}{l}\Delta \mathrm{E}_{S T}\left(\mathrm{E}_{\text {Open shell }}\right. \\
\text { singlet biradical state } \\
\text { Open shell triplelet state }\end{array}$ & $\begin{array}{l}\mathrm{y}_{0} \text { (biradical } \\
\text { yield) }\end{array}$ \\
\hline $\begin{array}{l}-2702.544887 \\
\text { a.u. }\end{array}$ & $\begin{array}{l}-2702.547566 \\
\text { a.u. }\end{array}$ & $\begin{array}{l}-2702.544254 \\
\text { a.u. }\end{array}$ & -0.003312 a.u. & $\begin{array}{l}0.48 \text { (uhf) } \\
0.09 \text { (udft) }\end{array}$ \\
\hline
\end{tabular}

Table S3. Calculation of the biradical character of $\mathbf{1 b}$

\begin{tabular}{|l|l|l|l|l|}
\hline $\begin{array}{l}E(\text { Close shell } \\
\text { state) }\end{array}$ & $\begin{array}{l}E \text { (Open shell } \\
\text { singlet biradical } \\
\text { state) }\end{array}$ & $\begin{array}{l}E \text { (Open shell } \\
\text { triplelet state) }\end{array}$ & $\begin{array}{l}\Delta \mathrm{E}_{S T}\left(\mathrm{E}_{\text {Open shell }}\right. \\
\text { singlet biradical state }-\mathrm{E} \\
\text { Open shell triplelet state }\end{array}$ & $\begin{array}{l}\mathrm{y}_{0} \text { (biradical } \\
\text { yield) }\end{array}$ \\
\hline $\begin{array}{l}-2702.548405 \\
\text { a.u. }\end{array}$ & $\begin{array}{l}-2702.549027 \\
\text { a.u. }\end{array}$ & $\begin{array}{l}-2702.540989 \\
\text { a.u. }\end{array}$ & -0.008038 a.u. & $\begin{array}{l}0.44 \text { (uhf) } \\
0.02 \text { (udft) }\end{array}$ \\
\hline
\end{tabular}


6.3 Visualization of the single occupied molecular orbitals

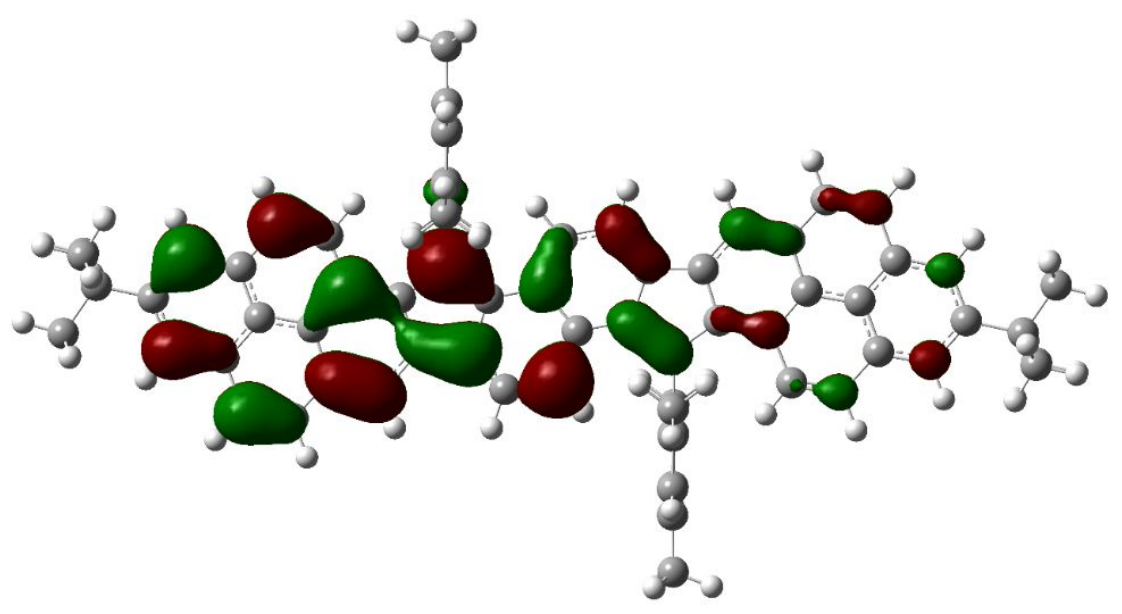

Figure S26. SOMO-a of 1a.

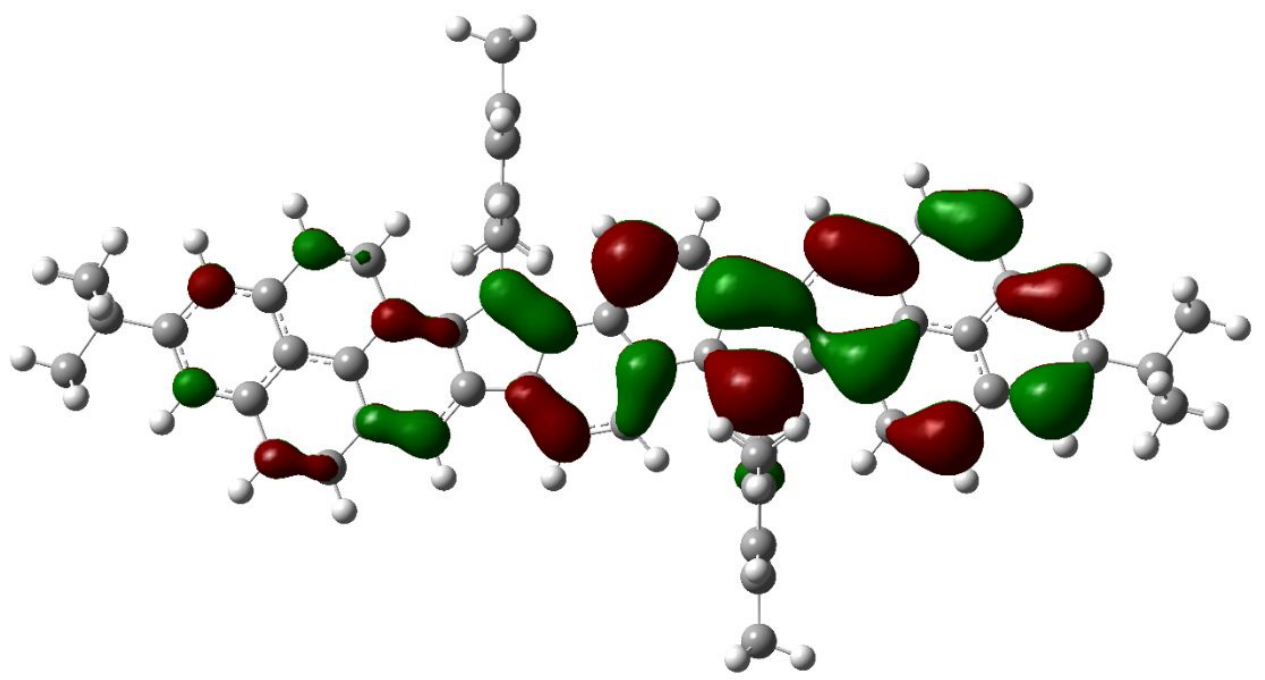

Figure S27. SOMO-b of 1a. 


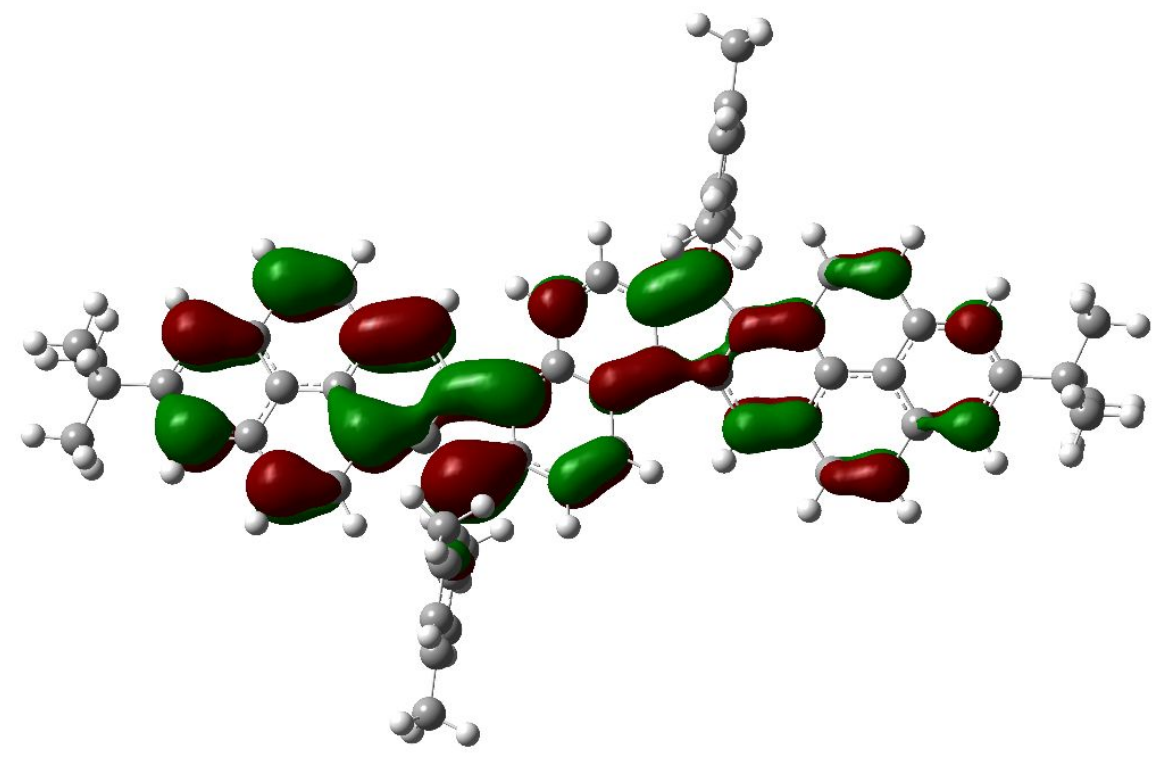

Figure S28. SOMO-a of $\mathbf{1 b .}$

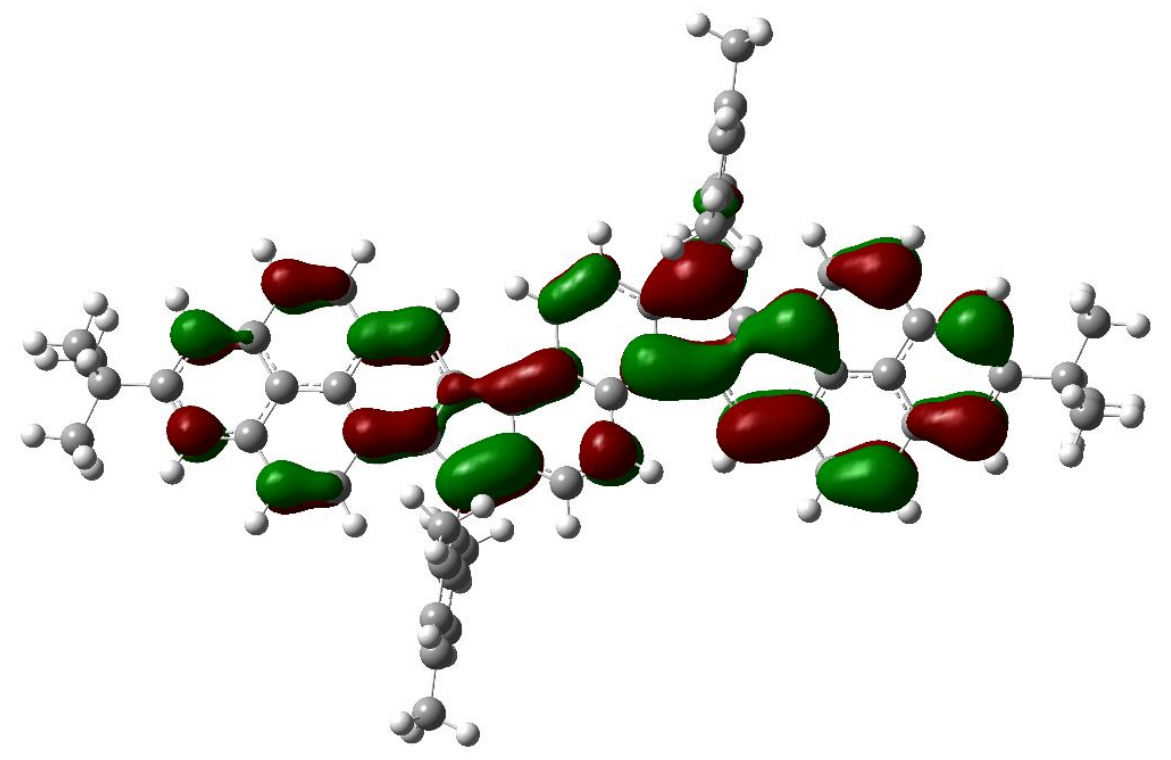

Figure S29. SOMO-b of $\mathbf{1 b}$. 


\subsection{Cartesian coordinates of all optimized structures}

1b closed-shell state:

$\mathrm{E}=-2702.548405$ a.u.

$\begin{array}{llll}\text { C } & -0.8230090 & -2.4321090 & 0.2678020\end{array}$

C $\quad-1.9821440 \quad-1.6071640 \quad 0.1829290$

$\begin{array}{llll}\text { C } & -1.8503800 & -0.1721240 & 0.1026270\end{array}$

$\begin{array}{llll}\text { C } & -0.5974400 & 0.4337360 & 0.1566770\end{array}$

$\begin{array}{llll}\text { C } & 0.5974420 & -0.4337260 & 0.1566710\end{array}$

$\begin{array}{llll}\text { C } & 0.4135260 & -1.8620080 & 0.2369880\end{array}$

$\begin{array}{llll}\text { C } & -0.4135220 & 1.8620170 & 0.2370040\end{array}$

$\begin{array}{llll}\text { C } & 0.8230140 & 2.4321170 & 0.2678160\end{array}$

$\begin{array}{llll}\text { C } & 1.9821480 & 1.6071720 & 0.1829330\end{array}$

$\begin{array}{llll}\text { C } & 1.8503820 & 0.1721330 & 0.1026220\end{array}$

$\begin{array}{llll}\text { C } & 3.2238050 & -0.3542540 & 0.0064580\end{array}$

$\begin{array}{llll}\text { C } & -3.2238050 & 0.3542620 & 0.0064710\end{array}$

$\begin{array}{llll}\text { C } & -4.1227990 & -0.7665170 & 0.0881510\end{array}$

$\begin{array}{llll}\text { C } & -5.5253300 & -0.5743520 & 0.0289100\end{array}$

$\begin{array}{llll}\text { C } & -6.0182550 & 0.7627970 & -0.1465650\end{array}$

$\begin{array}{llll}\text { C } & -5.1125690 & 1.8597520 & -0.2646320\end{array}$

$\begin{array}{llll}\text { C } & -3.7213650 & 1.6309690 & -0.1902560\end{array}$

$\begin{array}{llll}\text { C } & -6.4889290 & -1.6344920 & 0.1336630\end{array}$

$\begin{array}{llll}\text { C } & -7.8303090 & -1.3913010 & 0.0719840\end{array}$

$\begin{array}{llll}\text { C } & -8.3495970 & -0.0670780 & -0.1063340\end{array}$

$\begin{array}{llll}\text { C } & -7.4216910 & 1.0068970 & -0.2163810\end{array}$

$\begin{array}{llll}\text { C } & -9.7285350 & 0.1955910 & -0.1760080\end{array}$

$\begin{array}{llll}\text { C } & -10.2334570 & 1.4892510 & -0.3536100\end{array}$

$\begin{array}{llll}\text { C } & -9.3096340 & 2.5381850 & -0.4638180\end{array}$

$\begin{array}{llll}\text { C } & -7.9228160 & 2.3314280 & -0.4011280\end{array}$

$\begin{array}{llll}\text { C } & -6.9828930 & 3.4079480 & -0.5199680\end{array}$ 


$$
\begin{aligned}
& \text { C } \quad-5.6393120 \quad 3.1774240 \quad-0.4561380 \\
& \begin{array}{llll}
\text { C } & 4.1228010 & 0.7665220 & 0.0881390
\end{array} \\
& \begin{array}{llll}
\text { C } & 5.5253320 & 0.5743520 & 0.0288990
\end{array} \\
& \begin{array}{llll}
\text { C } & 6.0182520 & -0.7627960 & -0.1465890
\end{array} \\
& \begin{array}{llll}
\text { C } & 5.1125620 & -1.8597480 & -0.2646640
\end{array} \\
& \begin{array}{llll}
\text { C } & 3.7213600 & -1.6309620 & -0.1902790
\end{array} \\
& \begin{array}{llll}
\text { C } & 6.4889340 & 1.6344870 & 0.1336690
\end{array} \\
& \begin{array}{llll}
\text { C } & 7.8303140 & 1.3912930 & 0.0719870
\end{array} \\
& \begin{array}{llll}
\text { C } & 8.3495970 & 0.0670710 & -0.1063500
\end{array} \\
& \begin{array}{llll}
\text { C } & 7.4216880 & -1.0069000 & -0.2164100
\end{array} \\
& \begin{array}{llll}
\text { C } & 9.7285340 & -0.1956020 & -0.1760300
\end{array} \\
& \begin{array}{llll}
\text { C } & 10.2334520 & -1.4892620 & -0.3536510
\end{array} \\
& \begin{array}{llll}
\text { C } & 9.3096250 & -2.5381910 & -0.4638680
\end{array} \\
& \begin{array}{llll}
\text { C } & 7.9228080 & -2.3314310 & -0.4011710
\end{array} \\
& \begin{array}{llll}
\text { C } & 6.9828820 & -3.4079470 & -0.5200200
\end{array} \\
& \begin{array}{llll}
\text { C } & 5.6393010 & -3.1774200 & -0.4561840
\end{array} \\
& \begin{array}{llll}
\text { C } & 3.3289330 & 1.9744970 & 0.1970640
\end{array} \\
& \begin{array}{llll}
\text { C } & -3.3289280 & -1.9744910 & 0.1970660
\end{array} \\
& \begin{array}{llll}
\text { C } & -3.7908590 & -3.3851770 & 0.3064870
\end{array} \\
& \begin{array}{llll}
\text { C } & 3.7908650 & 3.3851830 & 0.3064770
\end{array} \\
& \text { C } \quad-4.0134110 \quad-3.9586950 \quad 1.5779030 \\
& \begin{array}{llll}
\text { C } & -4.4236440 & -5.2928230 & 1.6594780
\end{array} \\
& \begin{array}{llll}
\text { C } & -4.6137850 & -6.0795330 & 0.5195610
\end{array} \\
& \begin{array}{llll}
\text { C } & -4.3878300 & -5.4923940 & -0.7283200
\end{array} \\
& \begin{array}{llll}
\text { C } & -3.9772160 & -4.1615950 & -0.8577980
\end{array} \\
& \begin{array}{llll}
\text { C } & 3.9772710 & 4.1615770 & -0.8578130
\end{array} \\
& \begin{array}{llll}
\text { C } & 4.3878850 & 5.4923800 & -0.7283430
\end{array} \\
& \begin{array}{llll}
\text { C } & 4.6137910 & 6.0795400 & 0.5195340
\end{array} \\
& \begin{array}{llll}
\text { C } & 4.4236010 & 5.2928520 & 1.6594610
\end{array} \\
& \begin{array}{llll}
\text { C } & 4.0133680 & 3.9587260 & 1.5778930
\end{array}
\end{aligned}
$$




\begin{tabular}{|c|c|c|c|}
\hline & -3.7537930 & -3.5685210 & -2.2307130 \\
\hline $\mathrm{C}$ & -3.8283930 & -3.1455230 & 2.8393640 \\
\hline C & -5.0265430 & -7.5285820 & 0.6352730 \\
\hline $\mathrm{C}$ & 3.8282930 & 3.1455770 & 2.8393610 \\
\hline $\mathrm{C}$ & 3.7539000 & 3.5684810 & -2.2307270 \\
\hline $\mathrm{C}$ & 5.0265550 & 7.5285880 & 0.6352450 \\
\hline $\mathrm{C}$ & -11.7425970 & 1.7898400 & -0.4306670 \\
\hline $\mathrm{C}$ & -12.6031400 & 0.5197680 & -0.2894260 \\
\hline $\mathrm{C}$ & -12.1325420 & 2.7620050 & 0.7081770 \\
\hline $\mathrm{C}$ & -12.0690040 & 2.4415370 & -1.7954540 \\
\hline C & 11.7425910 & -1.7898560 & -0.4307080 \\
\hline $\mathrm{C}$ & 12.6031410 & -0.5197990 & -0.2893750 \\
\hline $\mathrm{C}$ & 12.1325110 & -2.7620960 & 0.7080790 \\
\hline $\mathrm{C}$ & 12.0690130 & -2.4414680 & -1.7955320 \\
\hline $\mathrm{H}$ & -0.9370730 & -3.5089330 & 0.3575150 \\
\hline $\mathrm{H}$ & 1.2870640 & -2.4918420 & 0.3300320 \\
\hline $\mathrm{H}$ & -1.2870570 & 2.4918520 & 0.3300570 \\
\hline $\mathrm{H}$ & 0.9370800 & 3.5089400 & 0.3575340 \\
\hline $\mathrm{H}$ & -3.0654420 & 2.4843160 & -0.3179120 \\
\hline $\mathrm{H}$ & -6.1375900 & -2.6495370 & 0.2695960 \\
\hline $\mathrm{H}$ & -8.5343310 & -2.2158720 & 0.1584000 \\
\hline $\mathrm{H}$ & -10.4051760 & -0.6477910 & -0.0875450 \\
\hline $\mathrm{H}$ & -9.6624320 & 3.5567830 & -0.6040600 \\
\hline $\mathrm{H}$ & -7.3623330 & 4.4168630 & -0.6630090 \\
\hline $\mathrm{H}$ & -4.9374020 & 4.0031640 & -0.5477620 \\
\hline $\mathrm{H}$ & 3.0654330 & -2.4843060 & -0.3179380 \\
\hline $\mathrm{H}$ & 6.1375990 & 2.6495310 & 0.2696190 \\
\hline $\mathrm{H}$ & 8.5343390 & 2.2158600 & 0.1584150 \\
\hline $\mathrm{H}$ & 10.4051780 & 0.6477760 & -0.0875580 \\
\hline $\mathrm{H}$ & 9.6624190 & -3.5567890 & -0.6041190 \\
\hline
\end{tabular}




\begin{tabular}{|c|c|c|c|}
\hline $\mathrm{H}$ & 7.3623180 & -4.4168620 & -0.6630720 \\
\hline $\mathrm{H}$ & 4.9373890 & -4.0031570 & -0.5478130 \\
\hline $\mathrm{H}$ & -4.5993030 & -5.7278060 & 2.6417210 \\
\hline $\mathrm{H}$ & -4.5364930 & -6.0848310 & -1.6292930 \\
\hline $\mathrm{H}$ & 4.5365830 & 6.0848000 & -1.6293200 \\
\hline $\mathrm{H}$ & 4.5992220 & 5.7278520 & 2.6417020 \\
\hline $\mathrm{H}$ & -3.9736490 & -4.3012060 & -3.0133590 \\
\hline $\mathrm{H}$ & -4.3920050 & -2.6928290 & -2.3979190 \\
\hline $\mathrm{H}$ & -2.7183040 & -3.2332640 & -2.3614740 \\
\hline $\mathrm{H}$ & -4.4849630 & -2.2671190 & 2.8511110 \\
\hline $\mathrm{H}$ & -4.0519500 & -3.7451290 & 3.7271480 \\
\hline $\mathrm{H}$ & -2.8017670 & -2.7726420 & 2.9318070 \\
\hline $\mathrm{H}$ & -5.6775080 & -7.6929470 & 1.5009150 \\
\hline $\mathrm{H}$ & -5.5604970 & -7.8660460 & -0.2593830 \\
\hline $\mathrm{H}$ & -4.1529510 & -8.1828790 & 0.7589330 \\
\hline $\mathrm{H}$ & 4.0518780 & 3.7451800 & 3.7271400 \\
\hline $\mathrm{H}$ & 2.8016440 & 2.7727580 & 2.9318020 \\
\hline $\mathrm{H}$ & 4.4848100 & 2.2671350 & 2.8511230 \\
\hline $\mathrm{H}$ & 2.7184270 & 3.2331830 & -2.3615060 \\
\hline $\mathrm{H}$ & 3.9737460 & 4.3011660 & -3.0133760 \\
\hline $\mathrm{H}$ & 4.3921500 & 2.6928110 & -2.3979100 \\
\hline $\mathrm{H}$ & 5.5600990 & 7.8661800 & -0.2596050 \\
\hline $\mathrm{H}$ & 4.1529980 & 8.1828410 & 0.7593860 \\
\hline $\mathrm{H}$ & 5.6779010 & 7.6928660 & 1.5006190 \\
\hline $\mathrm{H}$ & -13.6644720 & 0.7861680 & -0.3484660 \\
\hline $\mathrm{H}$ & -12.3994170 & -0.2026330 & -1.0879980 \\
\hline $\mathrm{H}$ & -12.4415980 & 0.0211640 & 0.6731370 \\
\hline $\mathrm{H}$ & -13.2047840 & 2.9894060 & 0.6646950 \\
\hline $\mathrm{H}$ & -11.5883880 & 3.7096740 & 0.6406690 \\
\hline $\mathrm{H}$ & -11.9176960 & 2.3226460 & 1.6889590 \\
\hline
\end{tabular}




$\begin{array}{llll}\mathrm{H} & -11.8085950 & 1.7708940 & -2.6221990 \\ \mathrm{H} & -13.1405210 & 2.6660910 & -1.8637570 \\ \mathrm{H} & -11.5224390 & 3.3791340 & -1.9418520 \\ \mathrm{H} & 13.6644710 & -0.7862000 & -0.3484420 \\ \mathrm{H} & 12.3994150 & 0.2026650 & -1.0878910 \\ \mathrm{H} & 12.4416070 & -0.0212680 & 0.6732280 \\ \mathrm{H} & 13.2047510 & -2.9895090 & 0.6645940 \\ \mathrm{H} & 11.5883440 & -3.7097530 & 0.6405060 \\ \mathrm{H} & 11.9176610 & -2.3227960 & 1.6888860 \\ \mathrm{H} & 11.8086130 & -1.7707740 & -2.6222390 \\ \mathrm{H} & 13.1405310 & -2.6660160 & -1.8638370 \\ \mathrm{H} & 11.5224520 & -3.3790570 & -1.9419960\end{array}$


1b open-shell singlet biradical state:

$\mathrm{E}=-2702.549027$ a.u.

C $\quad-0.82300900 \quad-2.43210900 \quad 0.26780200$

C $\quad-1.98214400 \quad-1.60716400 \quad 0.18292900$

C $\quad-1.85038000 \quad-0.17212400 \quad 0.10262700$

C $\quad-0.59744000 \quad 0.43373600 \quad 0.15667700$

C $\quad 0.59744200 \quad-0.43372600 \quad 0.15667100$

C $\quad 0.41352600 \quad-1.86200800 \quad 0.23698800$

C $\quad-0.41352200 \quad 1.86201700 \quad 0.23700400$

$\begin{array}{llll}\text { C } & 0.82301400 & 2.43211700 & 0.26781600\end{array}$

C $\quad 1.98214800 \quad 1.60717200 \quad 0.18293300$

C $\quad 1.85038200 \quad 0.17213300 \quad 0.10262200$

C $\quad 3.22380500 \quad-0.35425400 \quad 0.00645800$

C $\quad-3.22380500 \quad 0.35426200 \quad 0.00647100$

C $\quad-4.12279900 \quad-0.76651700 \quad 0.08815100$

C $\quad-5.52533000 \quad-0.57435200 \quad 0.02891000$

C $\quad-6.01825500 \quad 0.76279700 \quad-0.14656500$

C $\quad-5.11256900 \quad 1.85975200 \quad-0.26463200$

C $\quad-3.72136500 \quad 1.63096900 \quad-0.19025600$

C $\quad-6.48892900 \quad-1.63449200 \quad 0.13366300$

C $\quad-7.83030900 \quad-1.39130100 \quad 0.07198400$

C $\quad-8.34959700 \quad-0.06707800 \quad-0.10633400$

C $\quad-7.42169100 \quad 1.00689700 \quad-0.21638100$

C $\quad-9.72853500 \quad 0.19559100 \quad-0.17600800$

C $\quad-10.23345700 \quad 1.48925100 \quad-0.35361000$

C $\quad-9.30963400 \quad 2.53818500 \quad-0.46381800$

C $\quad-7.92281600 \quad 2.33142800 \quad-0.40112800$

C $\quad-6.98289300 \quad 3.40794800 \quad-0.51996800$

C $\quad-5.63931200 \quad 3.17742400 \quad-0.45613800$

C $\quad 4.12280100 \quad 0.76652200 \quad 0.08813900$ 


\begin{tabular}{|c|c|c|c|}
\hline $\mathrm{C}$ & 5.52533200 & 0.57435200 & 0.02889900 \\
\hline $\mathrm{C}$ & 6.01825200 & -0.76279600 & -0.14658900 \\
\hline $\mathrm{C}$ & 5.11256200 & -1.85974800 & -0.26466400 \\
\hline $\mathrm{C}$ & 3.72136000 & -1.63096200 & -0.19027900 \\
\hline $\mathrm{C}$ & 6.48893400 & 1.63448700 & 0.13366900 \\
\hline $\mathrm{C}$ & 7.83031400 & 1.39129300 & 0.07198700 \\
\hline $\mathrm{C}$ & 8.34959700 & 0.06707100 & -0.10635000 \\
\hline $\mathrm{C}$ & 7.42168800 & -1.00690000 & -0.21641000 \\
\hline $\mathrm{C}$ & 9.72853400 & -0.19560200 & -0.17603000 \\
\hline $\mathrm{C}$ & 10.23345200 & -1.48926200 & -0.35365100 \\
\hline $\mathrm{C}$ & 9.30962500 & -2.53819100 & -0.46386800 \\
\hline $\mathrm{C}$ & 7.92280800 & -2.33143100 & -0.40117100 \\
\hline $\mathrm{C}$ & 6.98288200 & -3.40794700 & -0.52002000 \\
\hline $\mathrm{C}$ & 5.63930100 & -3.17742000 & -0.45618400 \\
\hline $\mathrm{C}$ & 3.32893300 & 1.97449700 & 0.19706400 \\
\hline $\mathrm{C}$ & -3.32892800 & -1.97449100 & 0.19706600 \\
\hline $\mathrm{C}$ & -3.79085900 & -3.38517700 & 0.30648700 \\
\hline $\mathrm{C}$ & 3.79086500 & 3.38518300 & 0.30647700 \\
\hline $\mathrm{C}$ & -4.01341100 & -3.95869500 & 1.57790300 \\
\hline $\mathrm{C}$ & -4.42364400 & -5.29282300 & 1.65947800 \\
\hline $\mathrm{C}$ & -4.61378500 & -6.07953300 & 0.51956100 \\
\hline $\mathrm{C}$ & -4.38783000 & -5.49239400 & -0.72832000 \\
\hline $\mathrm{C}$ & -3.97721600 & -4.16159500 & -0.85779800 \\
\hline $\mathrm{C}$ & 3.97727100 & 4.16157700 & -0.85781300 \\
\hline $\mathrm{C}$ & 4.38788500 & 5.49238000 & -0.72834300 \\
\hline $\mathrm{C}$ & 4.61379100 & 6.07954000 & 0.51953400 \\
\hline $\mathrm{C}$ & 4.42360100 & 5.29285200 & 1.65946100 \\
\hline $\mathrm{C}$ & 4.01336800 & 3.95872600 & 1.57789300 \\
\hline $\mathrm{C}$ & -3.75379300 & -3.56852100 & -2.23071300 \\
\hline $\mathrm{C}$ & -3.82839300 & -3.14552300 & 2.83936400 \\
\hline
\end{tabular}




\begin{tabular}{|c|c|c|c|}
\hline $\mathrm{C}$ & -5.02654300 & -7.52858200 & 0.63527300 \\
\hline $\mathrm{C}$ & 3.82829300 & 3.14557700 & 2.83936100 \\
\hline $\mathrm{C}$ & 3.75390000 & 3.56848100 & -2.23072700 \\
\hline $\mathrm{C}$ & 5.02655500 & 7.52858800 & 0.63524500 \\
\hline $\mathrm{C}$ & -11.74259700 & 1.78984000 & -0.43066700 \\
\hline $\mathrm{C}$ & -12.60314000 & 0.51976800 & -0.28942600 \\
\hline $\mathrm{C}$ & -12.13254200 & 2.76200500 & 0.70817700 \\
\hline $\mathrm{C}$ & -12.06900400 & 2.44153700 & -1.79545400 \\
\hline $\mathrm{C}$ & 11.74259100 & -1.78985600 & -0.43070800 \\
\hline $\mathrm{C}$ & 12.60314100 & -0.51979900 & -0.28937500 \\
\hline $\mathrm{C}$ & 12.13251100 & -2.76209600 & 0.70807900 \\
\hline $\mathrm{C}$ & 12.06901300 & -2.44146800 & -1.79553200 \\
\hline $\mathrm{H}$ & -0.93707300 & -3.50893300 & 0.35751500 \\
\hline $\mathrm{H}$ & 1.28706400 & -2.49184200 & 0.33003200 \\
\hline $\mathrm{H}$ & -1.28705700 & 2.49185200 & 0.33005700 \\
\hline $\mathrm{H}$ & 0.93708000 & 3.50894000 & 0.35753400 \\
\hline $\mathrm{H}$ & -3.06544200 & 2.48431600 & -0.31791200 \\
\hline $\mathrm{H}$ & -6.13759000 & -2.64953700 & 0.26959600 \\
\hline $\mathrm{H}$ & -8.53433100 & -2.21587200 & 0.15840000 \\
\hline $\mathrm{H}$ & -10.40517600 & -0.64779100 & -0.08754500 \\
\hline $\mathrm{H}$ & -9.66243200 & 3.55678300 & -0.60406000 \\
\hline $\mathrm{H}$ & -7.36233300 & 4.41686300 & -0.66300900 \\
\hline $\mathrm{H}$ & -4.93740200 & 4.00316400 & -0.54776200 \\
\hline $\mathrm{H}$ & 3.06543300 & -2.48430600 & -0.31793800 \\
\hline $\mathrm{H}$ & 6.13759900 & 2.64953100 & 0.26961900 \\
\hline $\mathrm{H}$ & 8.53433900 & 2.21586000 & 0.15841500 \\
\hline $\mathrm{H}$ & 10.40517800 & 0.64777600 & -0.08755800 \\
\hline $\mathrm{H}$ & 9.66241900 & -3.55678900 & -0.60411900 \\
\hline $\mathrm{H}$ & 7.36231800 & -4.41686200 & -0.66307200 \\
\hline $\mathrm{H}$ & 4.93738900 & -4.00315700 & -0.54781300 \\
\hline & & & S37 \\
\hline
\end{tabular}




\begin{tabular}{|c|c|c|c|}
\hline $\mathrm{H}$ & -4.59930300 & -5.72780600 & 2.64172100 \\
\hline $\mathrm{H}$ & -4.53649300 & -6.08483100 & -1.62929300 \\
\hline $\mathrm{H}$ & 4.53658300 & 6.08480000 & -1.62932000 \\
\hline $\mathrm{H}$ & 4.59922200 & 5.72785200 & 2.64170200 \\
\hline $\mathrm{H}$ & -3.97364900 & -4.30120600 & -3.01335900 \\
\hline $\mathrm{H}$ & -4.39200500 & -2.69282900 & -2.39791900 \\
\hline H & -2.71830400 & -3.23326400 & -2.36147400 \\
\hline $\mathrm{H}$ & -4.48496300 & -2.26711900 & 2.85111100 \\
\hline $\mathrm{H}$ & -4.05195000 & -3.74512900 & 3.72714800 \\
\hline $\mathrm{H}$ & -2.80176700 & -2.77264200 & 2.93180700 \\
\hline $\mathrm{H}$ & -5.67750800 & -7.69294700 & 1.50091500 \\
\hline $\mathrm{H}$ & -5.56049700 & -7.86604600 & -0.25938300 \\
\hline $\mathrm{H}$ & -4.15295100 & -8.18287900 & 0.75893300 \\
\hline $\mathrm{H}$ & 4.05187800 & 3.74518000 & 3.72714000 \\
\hline $\mathrm{H}$ & 2.80164400 & 2.77275800 & 2.93180200 \\
\hline $\mathrm{H}$ & 4.48481000 & 2.26713500 & 2.85112300 \\
\hline $\mathrm{H}$ & 2.71842700 & 3.23318300 & -2.36150600 \\
\hline $\mathrm{H}$ & 3.97374600 & 4.30116600 & -3.01337600 \\
\hline $\mathrm{H}$ & 4.39215000 & 2.69281100 & -2.39791000 \\
\hline $\mathrm{H}$ & 5.56009900 & 7.86618000 & -0.25960500 \\
\hline $\mathrm{H}$ & 4.15299800 & 8.18284100 & 0.75938600 \\
\hline $\mathrm{H}$ & 5.67790100 & 7.69286600 & 1.50061900 \\
\hline $\mathrm{H}$ & -13.66447200 & 0.78616800 & -0.34846600 \\
\hline $\mathrm{H}$ & -12.39941700 & -0.20263300 & -1.08799800 \\
\hline $\mathrm{H}$ & -12.44159800 & 0.02116400 & 0.67313700 \\
\hline $\mathrm{H}$ & -13.20478400 & 2.98940600 & 0.66469500 \\
\hline $\mathrm{H}$ & -11.58838800 & 3.70967400 & 0.64066900 \\
\hline $\mathrm{H}$ & -11.91769600 & 2.32264600 & 1.68895900 \\
\hline $\mathrm{H}$ & -11.80859500 & 1.77089400 & -2.62219900 \\
\hline $\mathrm{H}$ & -13.14052100 & 2.66609100 & -1.86375700 \\
\hline
\end{tabular}




$\begin{array}{lrrr}\mathrm{H} & -11.52243900 & 3.37913400 & -1.94185200 \\ \mathrm{H} & 13.66447100 & -0.78620000 & -0.34844200 \\ \mathrm{H} & 12.39941500 & 0.20266500 & -1.08789100 \\ \mathrm{H} & 12.44160700 & -0.02126800 & 0.67322800 \\ \mathrm{H} & 13.20475100 & -2.98950900 & 0.66459400 \\ \mathrm{H} & 11.58834400 & -3.70975300 & 0.64050600 \\ \mathrm{H} & 11.91766100 & -2.32279600 & 1.68888600 \\ \mathrm{H} & 11.80861300 & -1.77077400 & -2.62223900 \\ \mathrm{H} & 13.14053100 & -2.66601600 & -1.86383700 \\ \mathrm{H} & 11.52245200 & -3.37905700 & -1.94199600\end{array}$


1b open-shell triplet state:

$\mathrm{E}=-2702.540989$ a.u.

$\mathrm{C}$

$\begin{array}{lll}0.83915200 & 2.41854200 & 0.22360700\end{array}$

$\begin{array}{llll}\mathrm{C} & 1.98369700 & 1.59533100 & 0.15954700\end{array}$

C $\quad 1.86864500 \quad 0.19182700 \quad 0.08205400$

C $\quad 0.58610200 \quad-0.42860300 \quad 0.11270300$

C $\quad-0.58610100 \quad 0.42860600 \quad 0.11270000$

C $\quad-0.40831200 \quad 1.84214500 \quad 0.18247100$

C $\quad 0.40831200 \quad-1.84214200 \quad 0.18248200$

C $\quad-0.83915200 \quad-2.41853900 \quad 0.22362000$

C $\quad-1.98369600 \quad-1.59532800 \quad 0.15955500$

C $\quad-1.86864400 \quad-0.19182500 \quad 0.08205300$

C $\quad-3.24188900 \quad 0.33168900 \quad-0.00254400$

C $\quad 3.24189000 \quad-0.33168800 \quad-0.00253900$

C $\quad 4.15096100 \quad 0.79908800 \quad 0.07876700$

C $\quad 5.56546900 \quad 0.59466900 \quad 0.02828000$

C $\quad 6.05078500 \quad-0.74238600 \quad-0.13636800$

C $\quad 5.13857200 \quad-1.83744800 \quad-0.25170200$

C $\quad 3.74166600 \quad-1.60365900 \quad-0.18610200$

C $\quad 6.52692100 \quad 1.64743100 \quad 0.13231400$

$\begin{array}{lllll}\text { C } & 7.87286300 & 1.39888700 & 0.07975400\end{array}$

C $\quad 8.38537600 \quad 0.07491600 \quad-0.08641300$

C $\quad 7.45268300 \quad-0.99470500 \quad-0.19541000$

C $\quad 9.76664300 \quad-0.19664100 \quad-0.14535100$

C $\quad 10.26530900-1.49261400 \quad-0.31057900$

C $\quad 9.33502400 \quad-2.53776600 \quad-0.41986000$

C $\quad 7.94817100 \quad-2.32311300 \quad-0.36772000$

C $\quad 7.00420300 \quad-3.39389700 \quad-0.48416800$

C $\quad 5.65798900 \quad-3.15468800 \quad-0.42947300$

C $\quad-4.15096000 \quad-0.79908600 \quad 0.07876700$ 


$\begin{array}{llll}\mathrm{C} & -5.56546900 & -0.59466700 & 0.02827800 \\ \mathrm{C} & -6.05078400 & 0.74238600 & -0.13638300 \\ \mathrm{C} & -5.13857200 & 1.83744800 & -0.25172400 \\ \mathrm{C} & -3.74166500 & 1.60366000 & -0.18611800 \\ \mathrm{C} & -6.52692000 & -1.64742900 & 0.13232100 \\ \mathrm{C} & -7.87286200 & -1.39888500 & 0.07975600 \\ \mathrm{C} & -8.38537500 & -0.07491700 & -0.08642600 \\ \mathrm{C} & -7.45268300 & 0.99470400 & -0.19543000 \\ \mathrm{C} & -9.76664200 & 0.19663900 & -0.14537100 \\ \mathrm{C} & -10.26530800 & 1.49261100 & -0.31061100 \\ \mathrm{C} & -9.33502400 & 2.53776200 & -0.41989900 \\ \mathrm{C} & -7.94817000 & 2.32311000 & -0.36775400 \\ \mathrm{C} & -4.02265500 & -3.98601400 & 1.54532800 \\ \mathrm{C} & -7.00420300 & 3.39389400 & -0.48420900 \\ \mathrm{C} & -4.77560700 & 3.56872200 & -2.26013000 \\ \mathrm{C} & -5.65798800 & 3.15468600 & -0.42950800 \\ \mathrm{C} & -3.37969200 & -1.98320900 & 0.17486100 \\ \mathrm{C} & 3.37969300 & 1.98321100 & 0.17485300 \\ \mathrm{C} & -3.81634800 & 3.40017500 & 0.27659700 \\ \mathrm{C} & -3.81634800 & -3.40017200 & 0.27660800 \\ \mathrm{C} & 4.02268000 & 3.98601300 & 1.54531400 \\ \mathrm{C} & 4.40212600 & 5.32948800 & 1.62071400 \\ \mathrm{C} & -3.57551900 & 6.11454700 & 0.47687600 \\ \mathrm{C} & -36372200 & 5.51642900 & -0.76830400 \\ \mathrm{C} & -3.17381700 & 2.80958300\end{array}$




\begin{tabular}{|c|c|c|c|}
\hline $\mathrm{C}$ & 4.95394900 & 7.57351300 & 0.58586200 \\
\hline $\mathrm{C}$ & -3.85329400 & -3.17382300 & 2.80959600 \\
\hline $\mathrm{C}$ & -3.77565600 & -3.56871100 & -2.26012100 \\
\hline $\mathrm{C}$ & -4.95395900 & -7.57350600 & 0.58588600 \\
\hline $\mathrm{C}$ & 11.77291700 & -1.80320500 & -0.37509200 \\
\hline $\mathrm{C}$ & 12.63996400 & -0.53732200 & -0.23654200 \\
\hline $\mathrm{C}$ & 12.14873900 & -2.76979100 & 0.77321000 \\
\hline $\mathrm{C}$ & 12.10467900 & -2.46643200 & -1.73300800 \\
\hline $\mathrm{C}$ & -11.77291700 & 1.80319800 & -0.37513800 \\
\hline $\mathrm{C}$ & -12.63996000 & 0.53730400 & -0.23666600 \\
\hline $\mathrm{C}$ & -12.14876800 & 2.76972800 & 0.77320100 \\
\hline $\mathrm{C}$ & -12.10465200 & 2.46648700 & -1.73303100 \\
\hline $\mathrm{H}$ & 0.94908400 & 3.49665500 & 0.30215900 \\
\hline $\mathrm{H}$ & -1.27898100 & 2.47947400 & 0.25429100 \\
\hline $\mathrm{H}$ & 1.27898100 & -2.47947000 & 0.25430500 \\
\hline $\mathrm{H}$ & -0.94908300 & -3.49665100 & 0.30217700 \\
\hline $\mathrm{H}$ & 3.08825600 & -2.46008900 & -0.30712600 \\
\hline $\mathrm{H}$ & 6.17894200 & 2.66519900 & 0.25928500 \\
\hline $\mathrm{H}$ & 8.57817700 & 2.22241500 & 0.16508500 \\
\hline $\mathrm{H}$ & 10.44659300 & 0.64415600 & -0.05771400 \\
\hline $\mathrm{H}$ & 9.68305200 & -3.55925000 & -0.55054700 \\
\hline $\mathrm{H}$ & 7.37752900 & -4.40636000 & -0.61753700 \\
\hline $\mathrm{H}$ & 4.95365900 & -3.97857000 & -0.51896500 \\
\hline $\mathrm{H}$ & -3.08825600 & 2.46008900 & -0.30714800 \\
\hline $\mathrm{H}$ & -6.17894100 & -2.66519600 & 0.25930400 \\
\hline $\mathrm{H}$ & -8.57817600 & -2.22241300 & 0.16509400 \\
\hline $\mathrm{H}$ & -10.44659200 & -0.64415800 & -0.05772900 \\
\hline $\mathrm{H}$ & -9.68305100 & 3.55924500 & -0.55059900 \\
\hline $\mathrm{H}$ & -7.37752900 & 4.40635500 & -0.61758800 \\
\hline $\mathrm{H}$ & -4.95365800 & 3.97856700 & -0.51900600 \\
\hline
\end{tabular}

\section{S42}




\begin{tabular}{|c|c|c|c|}
\hline $\mathrm{H}$ & 4.56520000 & 5.77381400 & 2.60099800 \\
\hline $\mathrm{H}$ & 4.49752300 & 6.10812400 & -1.67211900 \\
\hline $\mathrm{H}$ & -4.49756800 & -6.10811300 & -1.67210300 \\
\hline $\mathrm{H}$ & -4.56515700 & -5.77381700 & 2.60101600 \\
\hline $\mathrm{H}$ & 3.92629700 & 4.31504100 & -3.04632600 \\
\hline $\mathrm{H}$ & 4.47189000 & 2.74126300 & -2.44167700 \\
\hline $\mathrm{H}$ & 2.76437300 & 3.15972200 & -2.36941500 \\
\hline $\mathrm{H}$ & 4.55288400 & 2.32974600 & 2.84125400 \\
\hline $\mathrm{H}$ & 4.02840800 & 3.78997400 & 3.69699200 \\
\hline $\mathrm{H}$ & 2.84493600 & 2.75041100 & 2.88440500 \\
\hline $\mathrm{H}$ & 5.60261900 & 7.75678900 & 1.44946500 \\
\hline $\mathrm{H}$ & 5.47806500 & 7.91984900 & -0.31123300 \\
\hline $\mathrm{H}$ & 4.06547100 & 8.20762300 & 0.70875900 \\
\hline $\mathrm{H}$ & -4.02835400 & -3.78997800 & 3.69700600 \\
\hline $\mathrm{H}$ & -2.84487600 & -2.75043300 & 2.88440600 \\
\hline $\mathrm{H}$ & -4.55281800 & -2.32974200 & 2.84127600 \\
\hline $\mathrm{H}$ & -2.76442600 & -3.15970500 & -2.36942100 \\
\hline $\mathrm{H}$ & -3.92635400 & -4.31503000 & -3.04631400 \\
\hline $\mathrm{H}$ & -4.47194800 & -2.74125600 & -2.44165600 \\
\hline $\mathrm{H}$ & -5.47784300 & -7.91991200 & -0.31131700 \\
\hline $\mathrm{H}$ & -4.06550400 & -8.20759500 & 0.70905800 \\
\hline $\mathrm{H}$ & -5.60284700 & -7.75673100 & 1.44933700 \\
\hline $\mathrm{H}$ & 13.70005800 & -0.81037400 & -0.28612800 \\
\hline $\mathrm{H}$ & 12.44628600 & 0.18068100 & -1.04156600 \\
\hline $\mathrm{H}$ & 12.47453800 & -0.03111500 & 0.72138200 \\
\hline $\mathrm{H}$ & 13.21979800 & -3.00406700 & 0.73893200 \\
\hline $\mathrm{H}$ & 11.59940800 & -3.71469600 & 0.70842600 \\
\hline $\mathrm{H}$ & 11.92967300 & -2.32236000 & 1.74938800 \\
\hline $\mathrm{H}$ & 11.85516500 & -1.79962600 & -2.56618200 \\
\hline $\mathrm{H}$ & 13.17501900 & -2.69900100 & -1.79181100 \\
\hline
\end{tabular}




$\begin{array}{lrrr}\mathrm{H} & 11.55271500 & -3.40124500 & -1.87710800 \\ \mathrm{H} & -13.70005500 & 0.81035800 & -0.28623100 \\ \mathrm{H} & -12.44628500 & -0.18064600 & -1.04173700 \\ \mathrm{H} & -12.47452900 & 0.03103600 & 0.72122500 \\ \mathrm{H} & -13.21982800 & 3.00399600 & 0.73891400 \\ \mathrm{H} & -11.59944600 & 3.71464100 & 0.70847200 \\ \mathrm{H} & -11.92971700 & 2.32225400 & 1.74936300 \\ \mathrm{H} & -11.85512300 & 1.79971900 & -2.56623000 \\ \mathrm{H} & -13.17499100 & 2.69906000 & -1.79184400 \\ \mathrm{H} & -11.55268400 & 3.40130600 & -1.87707700\end{array}$


1a closed-shell state:

$\mathrm{E}=-2702.544887$ a.u.

C $\quad-1.27403900 \quad-2.23049800 \quad 0.00001200$

$\begin{array}{llll}\text { C } & -2.24646000 & -1.26305300 & 0.00000600\end{array}$

$\begin{array}{llll}\text { C } & -1.90592000 & 0.13948500 & 0.00000500\end{array}$

$\begin{array}{llll}\text { C } & -0.50606000 & 0.53425200 & 0.00001800\end{array}$

$\begin{array}{llll}\text { C } & 0.50606200 & -0.53424900 & 0.00001800\end{array}$

$\begin{array}{llll}\text { C } & 0.08972900 & -1.86390600 & 0.00001500\end{array}$

$\begin{array}{llll}\text { C } & -0.08972700 & 1.86390900 & 0.00003200\end{array}$

$\begin{array}{llll}\text { C } & 1.27404200 & 2.23050000 & 0.00003600\end{array}$

$\begin{array}{llll}\text { C } & 2.24646200 & 1.26305500 & 0.00002900\end{array}$

$\begin{array}{llll}\text { C } & 1.90592100 & -0.13948200 & 0.00002300\end{array}$

$\begin{array}{llll}\text { C } & 3.69786700 & 1.37101400 & 0.00002900\end{array}$

C $\quad-3.69786600 \quad-1.37101200 \quad 0.00000000$

$\begin{array}{llll}\text { C } & -4.51359300 & -2.48327100 & 0.00000700\end{array}$

$\begin{array}{llll}\text { C } & -5.91542200 & -2.32671300 & 0.00000300\end{array}$

C $\quad-6.47638500 \quad-1.00944200 \quad-0.00001600$

$\begin{array}{llll}\text { C } & -5.63225400 & 0.15621800 & -0.00002900\end{array}$

$\begin{array}{llll}\text { C } & -4.22575100 & -0.04129400 & -0.00001300\end{array}$

$\begin{array}{llll}\text { C } & -6.79160900 & -3.45760500 & 0.00001900\end{array}$

$\begin{array}{llll}\text { C } & -8.14859900 & -3.31074800 & 0.00001600\end{array}$

$\begin{array}{llll}\text { C } & -8.74828000 & -2.00962000 & -0.00000300\end{array}$

C $\quad-7.89651200 \quad-0.86505800 \quad-0.00002100$

$\begin{array}{llll}\text { C } & -10.14537300 & -1.83602800 & -0.00000400\end{array}$

$\begin{array}{llll}\text { C } & -10.74109000 & -0.57162000 & -0.00002000\end{array}$

$\begin{array}{llll}\text { C } & -9.89182200 & 0.54619500 & -0.00004400\end{array}$

$\begin{array}{llll}\text { C } & -8.49510100 & 0.42961500 & -0.00004400\end{array}$

C $\quad-7.62948400 \quad 1.57178300 \quad-0.00006500$

C $\quad-6.27140100 \quad 1.44300300 \quad-0.00005800$

$\begin{array}{llll}\text { C } & 4.51359500 & 2.48327200 & 0.00002700\end{array}$ 


\begin{tabular}{|c|c|c|c|}
\hline $\mathrm{C}$ & 5.91542300 & 2.32671300 & 0.00002000 \\
\hline $\mathrm{C}$ & 6.47638600 & 1.00944100 & 0.00001700 \\
\hline $\mathrm{C}$ & 5.63225300 & -0.15621800 & 0.00002100 \\
\hline $\mathrm{C}$ & 4.22575200 & 0.04129600 & 0.00002400 \\
\hline $\mathrm{C}$ & 6.79161100 & 3.45760500 & 0.00001500 \\
\hline $\mathrm{C}$ & 8.14860200 & 3.31074600 & 0.00000500 \\
\hline $\mathrm{C}$ & 8.74828200 & 2.00961700 & 0.00000200 \\
\hline $\mathrm{C}$ & 7.89651200 & 0.86505600 & 0.00001000 \\
\hline $\mathrm{C}$ & 10.14537400 & 1.83602400 & -0.00000900 \\
\hline $\mathrm{C}$ & 10.74108900 & 0.57161600 & -0.00000600 \\
\hline $\mathrm{C}$ & 9.89182000 & -0.54619800 & -0.00000100 \\
\hline $\mathrm{C}$ & 8.49510000 & -0.42961700 & 0.00000700 \\
\hline $\mathrm{C}$ & 7.62948200 & -1.57178400 & 0.00001400 \\
\hline $\mathrm{C}$ & 6.27140000 & -1.44300200 & 0.00002200 \\
\hline $\mathrm{C}$ & -3.10302900 & 0.88395600 & -0.00000800 \\
\hline $\mathrm{C}$ & 3.10303000 & -0.88395300 & 0.00002100 \\
\hline $\mathrm{C}$ & 3.21472500 & -2.36790300 & 0.00001800 \\
\hline $\mathrm{C}$ & -3.21472500 & 2.36790500 & -0.00000100 \\
\hline $\mathrm{C}$ & -3.26293200 & 3.07348700 & -1.22238200 \\
\hline $\mathrm{C}$ & -3.36131300 & 4.46830100 & -1.19847700 \\
\hline $\mathrm{C}$ & -3.41504800 & 5.18627200 & 0.00000700 \\
\hline $\mathrm{C}$ & -3.36144500 & 4.46829000 & 1.19847000 \\
\hline $\mathrm{C}$ & -3.26306300 & 3.07345900 & 1.22236900 \\
\hline $\mathrm{C}$ & 3.26297600 & -3.07347500 & 1.22239400 \\
\hline $\mathrm{C}$ & 3.36135700 & -4.46829400 & 1.19849100 \\
\hline $\mathrm{C}$ & 3.41504500 & -5.18627000 & 0.00001500 \\
\hline $\mathrm{C}$ & 3.36139800 & -4.46829000 & -1.19845600 \\
\hline $\mathrm{C}$ & 3.26301700 & -3.07346600 & -1.22235600 \\
\hline $\mathrm{C}$ & 3.20367400 & -2.33891900 & -2.54225400 \\
\hline $\mathrm{C}$ & 3.20358600 & -2.33893000 & 2.54229100 \\
\hline
\end{tabular}




\begin{tabular}{|c|c|c|c|}
\hline $\mathrm{C}$ & 3.55319000 & -6.69112300 & 0.00002300 \\
\hline $\mathrm{C}$ & -3.20349100 & 2.33894200 & -2.54227600 \\
\hline $\mathrm{C}$ & -3.20376900 & 2.33891200 & 2.54226900 \\
\hline $\mathrm{C}$ & -3.55319400 & 6.69112600 & -0.00002400 \\
\hline $\mathrm{C}$ & -12.26800800 & -0.36904600 & -0.00002200 \\
\hline $\mathrm{C}$ & -13.03760500 & -1.70364400 & -0.00004100 \\
\hline $\mathrm{C}$ & -12.68156200 & 0.42434200 & 1.26233600 \\
\hline $\mathrm{C}$ & -12.68154900 & 0.42437300 & -1.26236300 \\
\hline $\mathrm{C}$ & 12.26800800 & 0.36904000 & -0.00002200 \\
\hline $\mathrm{C}$ & 13.03760600 & 1.70363700 & -0.00014300 \\
\hline $\mathrm{C}$ & 12.68158400 & -0.42426500 & 1.26238000 \\
\hline $\mathrm{C}$ & 12.68152400 & -0.42446100 & -1.26232000 \\
\hline $\mathrm{H}$ & -1.53619400 & -3.28587000 & 0.00001300 \\
\hline $\mathrm{H}$ & 0.83015200 & -2.65236200 & 0.00001800 \\
\hline $\mathrm{H}$ & -0.83014900 & 2.65236500 & 0.00004200 \\
\hline $\mathrm{H}$ & 1.53619600 & 3.28587200 & 0.00004600 \\
\hline $\mathrm{H}$ & -4.09259700 & -3.48641300 & 0.00001900 \\
\hline $\mathrm{H}$ & -6.34773500 & -4.45049900 & 0.00003300 \\
\hline $\mathrm{H}$ & -8.79741100 & -4.18336100 & 0.00003000 \\
\hline $\mathrm{H}$ & -10.75982600 & -2.73025100 & 0.00001300 \\
\hline $\mathrm{H}$ & -10.31581800 & 1.54716500 & -0.00006300 \\
\hline $\mathrm{H}$ & -8.07913400 & 2.56214900 & -0.00008700 \\
\hline $\mathrm{H}$ & -5.65613700 & 2.33316900 & -0.00007500 \\
\hline $\mathrm{H}$ & 4.09260000 & 3.48641400 & 0.00003100 \\
\hline $\mathrm{H}$ & 6.34773900 & 4.45049900 & 0.00001700 \\
\hline $\mathrm{H}$ & 8.79741400 & 4.18335900 & 0.00000000 \\
\hline $\mathrm{H}$ & 10.75982800 & 2.73024700 & -0.00001500 \\
\hline $\mathrm{H}$ & 10.31581500 & -1.54716800 & -0.00000600 \\
\hline $\mathrm{H}$ & 8.07913100 & -2.56215000 & 0.00001600 \\
\hline \multirow[t]{2}{*}{$\mathrm{H}$} & 5.65613500 & -2.33316700 & 0.00002800 \\
\hline & & & S47 \\
\hline
\end{tabular}




\begin{tabular}{|c|c|c|c|}
\hline $\mathrm{H}$ & -3.39710000 & 5.00755500 & -2.14337700 \\
\hline $\mathrm{H}$ & -3.39734100 & 5.00753300 & 2.14336900 \\
\hline $\mathrm{H}$ & 3.39718000 & -5.00754500 & 2.14339100 \\
\hline $\mathrm{H}$ & 3.39725500 & -5.00753700 & -2.14335600 \\
\hline $\mathrm{H}$ & 3.26436200 & -3.03810400 & -3.38200100 \\
\hline $\mathrm{H}$ & 2.27191700 & -1.76954200 & -2.64226800 \\
\hline $\mathrm{H}$ & 4.02631900 & -1.62073300 & -2.63910600 \\
\hline $\mathrm{H}$ & 2.27181300 & -1.76957500 & 2.64228400 \\
\hline $\mathrm{H}$ & 3.26427200 & -3.03811400 & 3.38203900 \\
\hline $\mathrm{H}$ & 4.02621100 & -1.62072400 & 2.63916100 \\
\hline H & 3.08824000 & -7.13657700 & 0.88609000 \\
\hline $\mathrm{H}$ & 3.08926800 & -7.13648700 & -0.88662300 \\
\hline $\mathrm{H}$ & 4.60886500 & -6.99469000 & 0.00063100 \\
\hline $\mathrm{H}$ & -4.02610200 & 1.62072300 & -2.63917100 \\
\hline $\mathrm{H}$ & -3.26416200 & 3.03812500 & -3.38202700 \\
\hline $\mathrm{H}$ & -2.27170500 & 1.76960300 & -2.64224000 \\
\hline $\mathrm{H}$ & -3.26447300 & 3.03809800 & 3.38201400 \\
\hline $\mathrm{H}$ & -4.02642800 & 1.62073800 & 2.63909700 \\
\hline $\mathrm{H}$ & -2.27202300 & 1.76952100 & 2.64231200 \\
\hline $\mathrm{H}$ & -3.08697000 & 7.13669100 & -0.88537000 \\
\hline $\mathrm{H}$ & -4.60886600 & 6.99469200 & -0.00213700 \\
\hline $\mathrm{H}$ & -3.09054800 & 7.13637900 & 0.88734000 \\
\hline $\mathrm{H}$ & -14.11589200 & -1.50782300 & -0.00002200 \\
\hline $\mathrm{H}$ & -12.81213600 & -2.30510700 & -0.88803800 \\
\hline $\mathrm{H}$ & -12.81211000 & -2.30514600 & 0.88792400 \\
\hline $\mathrm{H}$ & -13.76700700 & 0.58223900 & 1.27491700 \\
\hline $\mathrm{H}$ & -12.20142300 & 1.40769300 & 1.30190400 \\
\hline $\mathrm{H}$ & -12.40610300 & -0.11848800 & 2.17370700 \\
\hline $\mathrm{H}$ & -12.40610800 & -0.11844800 & -2.17374500 \\
\hline $\mathrm{H}$ & -13.76699100 & 0.58229700 & -1.27493600 \\
\hline
\end{tabular}




$\begin{array}{lrrr}\mathrm{H} & -12.20138500 & 1.40771200 & -1.30191500 \\ \mathrm{H} & 14.11589200 & 1.50781500 & -0.00012200 \\ \mathrm{H} & 12.81212800 & 2.30503900 & -0.88818000 \\ \mathrm{H} & 12.81212100 & 2.30520200 & 0.88778100 \\ \mathrm{H} & 13.76703000 & -0.58215900 & 1.27495200 \\ \mathrm{H} & 12.20144800 & -1.40761400 & 1.30201900 \\ \mathrm{H} & 12.40614000 & 0.11862400 & 2.17372000 \\ \mathrm{H} & 12.40606900 & 0.11830100 & -2.17373200 \\ \mathrm{H} & 13.76696500 & -0.58239100 & -1.27490100 \\ \mathrm{H} & 12.20135500 & -1.40780200 & -1.30180000\end{array}$


1a open-shell singlet biradical state:

$\mathrm{E}=-2702.547566$ a.u.

$\begin{array}{llll}\text { C } & -1.27403900 & -2.23049800 & 0.00001200\end{array}$

C $\quad-2.24646000 \quad-1.26305300 \quad 0.00000600$

$\begin{array}{llll}\text { C } & -1.90592000 & 0.13948500 & 0.00000500\end{array}$

C $\quad-0.50606000 \quad 0.53425200 \quad 0.00001800$

C $\quad 0.50606200 \quad-0.53424900 \quad 0.00001800$

C $\quad 0.08972900 \quad-1.86390600 \quad 0.00001500$

C $\quad-0.08972700 \quad 1.86390900 \quad 0.00003200$

$\begin{array}{llll}\mathrm{C} & 1.27404200 & 2.23050000 & 0.00003600\end{array}$

C $\quad 2.24646200 \quad 1.26305500 \quad 0.00002900$

C $\quad 1.90592100 \quad-0.13948200 \quad 0.00002300$

C $\quad 3.69786700 \quad 1.37101400 \quad 0.00002900$

C $\quad-3.69786600 \quad-1.37101200 \quad 0.00000000$

C $\quad-4.51359300 \quad-2.48327100 \quad 0.00000700$

C $\quad-5.91542200 \quad-2.32671300 \quad 0.00000300$

C $\quad-6.47638500-1.00944200 \quad-0.00001600$

C $\quad-5.63225400 \quad 0.15621800 \quad-0.00002900$

C $\quad-4.22575100 \quad-0.04129400 \quad-0.00001300$

C $\quad-6.79160900 \quad-3.45760500 \quad 0.00001900$

C $\quad-8.14859900 \quad-3.31074800 \quad 0.00001600$

C $\quad-8.74828000 \quad-2.00962000 \quad-0.00000300$

C $\quad-7.89651200 \quad-0.86505800 \quad-0.00002100$

C $\quad-10.14537300 \quad-1.83602800 \quad-0.00000400$

C $\quad-10.74109000 \quad-0.57162000-0.00002000$

C $\quad-9.89182200 \quad 0.54619500 \quad-0.00004400$

C $\quad-8.49510100 \quad 0.42961500 \quad-0.00004400$

C $\quad-7.62948400 \quad 1.57178300 \quad-0.00006500$

C $\quad-6.27140100 \quad 1.44300300 \quad-0.00005800$

$\begin{array}{llll}\text { C } & 4.51359500 & 2.48327200 & 0.00002700\end{array}$ 


\begin{tabular}{|c|c|c|c|}
\hline $\mathrm{C}$ & 5.91542300 & 2.32671300 & 0.00002000 \\
\hline $\mathrm{C}$ & 6.47638600 & 1.00944100 & 0.00001700 \\
\hline $\mathrm{C}$ & 5.63225300 & -0.15621800 & 0.00002100 \\
\hline $\mathrm{C}$ & 4.22575200 & 0.04129600 & 0.00002400 \\
\hline $\mathrm{C}$ & 6.79161100 & 3.45760500 & 0.00001500 \\
\hline $\mathrm{C}$ & 8.14860200 & 3.31074600 & 0.00000500 \\
\hline $\mathrm{C}$ & 8.74828200 & 2.00961700 & 0.00000200 \\
\hline $\mathrm{C}$ & 7.89651200 & 0.86505600 & 0.00001000 \\
\hline $\mathrm{C}$ & 10.14537400 & 1.83602400 & -0.00000900 \\
\hline $\mathrm{C}$ & 10.74108900 & 0.57161600 & -0.00000600 \\
\hline $\mathrm{C}$ & 9.89182000 & -0.54619800 & -0.00000100 \\
\hline $\mathrm{C}$ & 8.49510000 & -0.42961700 & 0.00000700 \\
\hline $\mathrm{C}$ & 7.62948200 & -1.57178400 & 0.00001400 \\
\hline $\mathrm{C}$ & 6.27140000 & -1.44300200 & 0.00002200 \\
\hline $\mathrm{C}$ & -3.10302900 & 0.88395600 & -0.00000800 \\
\hline $\mathrm{C}$ & 3.10303000 & -0.88395300 & 0.00002100 \\
\hline $\mathrm{C}$ & 3.21472500 & -2.36790300 & 0.00001800 \\
\hline $\mathrm{C}$ & -3.21472500 & 2.36790500 & -0.00000100 \\
\hline $\mathrm{C}$ & -3.26293200 & 3.07348700 & -1.22238200 \\
\hline $\mathrm{C}$ & -3.36131300 & 4.46830100 & -1.19847700 \\
\hline $\mathrm{C}$ & -3.41504800 & 5.18627200 & 0.00000700 \\
\hline $\mathrm{C}$ & -3.36144500 & 4.46829000 & 1.19847000 \\
\hline $\mathrm{C}$ & -3.26306300 & 3.07345900 & 1.22236900 \\
\hline $\mathrm{C}$ & 3.26297600 & -3.07347500 & 1.22239400 \\
\hline $\mathrm{C}$ & 3.36135700 & -4.46829400 & 1.19849100 \\
\hline $\mathrm{C}$ & 3.41504500 & -5.18627000 & 0.00001500 \\
\hline $\mathrm{C}$ & 3.36139800 & -4.46829000 & -1.19845600 \\
\hline $\mathrm{C}$ & 3.26301700 & -3.07346600 & -1.22235600 \\
\hline $\mathrm{C}$ & 3.20367400 & -2.33891900 & -2.54225400 \\
\hline $\mathrm{C}$ & 3.20358600 & -2.33893000 & 2.54229100 \\
\hline
\end{tabular}




\begin{tabular}{|c|c|c|c|}
\hline $\mathrm{C}$ & 3.55319000 & -6.69112300 & 0.00002300 \\
\hline $\mathrm{C}$ & -3.20349100 & 2.33894200 & -2.54227600 \\
\hline $\mathrm{C}$ & -3.20376900 & 2.33891200 & 2.54226900 \\
\hline $\mathrm{C}$ & -3.55319400 & 6.69112600 & -0.00002400 \\
\hline $\mathrm{C}$ & -12.26800800 & -0.36904600 & -0.00002200 \\
\hline $\mathrm{C}$ & -13.03760500 & -1.70364400 & -0.00004100 \\
\hline $\mathrm{C}$ & -12.68156200 & 0.42434200 & 1.26233600 \\
\hline $\mathrm{C}$ & -12.68154900 & 0.42437300 & -1.26236300 \\
\hline $\mathrm{C}$ & 12.26800800 & 0.36904000 & -0.00002200 \\
\hline $\mathrm{C}$ & 13.03760600 & 1.70363700 & -0.00014300 \\
\hline $\mathrm{C}$ & 12.68158400 & -0.42426500 & 1.26238000 \\
\hline $\mathrm{C}$ & 12.68152400 & -0.42446100 & -1.26232000 \\
\hline $\mathrm{H}$ & -1.53619400 & -3.28587000 & 0.00001300 \\
\hline $\mathrm{H}$ & 0.83015200 & -2.65236200 & 0.00001800 \\
\hline $\mathrm{H}$ & -0.83014900 & 2.65236500 & 0.00004200 \\
\hline $\mathrm{H}$ & 1.53619600 & 3.28587200 & 0.00004600 \\
\hline $\mathrm{H}$ & -4.09259700 & -3.48641300 & 0.00001900 \\
\hline $\mathrm{H}$ & -6.34773500 & -4.45049900 & 0.00003300 \\
\hline $\mathrm{H}$ & -8.79741100 & -4.18336100 & 0.00003000 \\
\hline $\mathrm{H}$ & -10.75982600 & -2.73025100 & 0.00001300 \\
\hline $\mathrm{H}$ & -10.31581800 & 1.54716500 & -0.00006300 \\
\hline $\mathrm{H}$ & -8.07913400 & 2.56214900 & -0.00008700 \\
\hline $\mathrm{H}$ & -5.65613700 & 2.33316900 & -0.00007500 \\
\hline $\mathrm{H}$ & 4.09260000 & 3.48641400 & 0.00003100 \\
\hline $\mathrm{H}$ & 6.34773900 & 4.45049900 & 0.00001700 \\
\hline $\mathrm{H}$ & 8.79741400 & 4.18335900 & 0.00000000 \\
\hline $\mathrm{H}$ & 10.75982800 & 2.73024700 & -0.00001500 \\
\hline $\mathrm{H}$ & 10.31581500 & -1.54716800 & -0.00000600 \\
\hline $\mathrm{H}$ & 8.07913100 & -2.56215000 & 0.00001600 \\
\hline $\mathrm{H}$ & 5.65613500 & -2.33316700 & 0.00002800 \\
\hline
\end{tabular}




\begin{tabular}{|c|c|c|c|}
\hline $\mathrm{H}$ & -3.39710000 & 5.00755500 & -2.14337700 \\
\hline $\mathrm{H}$ & -3.39734100 & 5.00753300 & 2.14336900 \\
\hline $\mathrm{H}$ & 3.39718000 & -5.00754500 & 2.14339100 \\
\hline $\mathrm{H}$ & 3.39725500 & -5.00753700 & -2.14335600 \\
\hline $\mathrm{H}$ & 3.26436200 & -3.03810400 & -3.38200100 \\
\hline $\mathrm{H}$ & 2.27191700 & -1.76954200 & -2.64226800 \\
\hline $\mathrm{H}$ & 4.02631900 & -1.62073300 & -2.63910600 \\
\hline $\mathrm{H}$ & 2.27181300 & -1.76957500 & 2.64228400 \\
\hline $\mathrm{H}$ & 3.26427200 & -3.03811400 & 3.38203900 \\
\hline $\mathrm{H}$ & 4.02621100 & -1.62072400 & 2.63916100 \\
\hline H & 3.08824000 & -7.13657700 & 0.88609000 \\
\hline $\mathrm{H}$ & 3.08926800 & -7.13648700 & -0.88662300 \\
\hline $\mathrm{H}$ & 4.60886500 & -6.99469000 & 0.00063100 \\
\hline $\mathrm{H}$ & -4.02610200 & 1.62072300 & -2.63917100 \\
\hline $\mathrm{H}$ & -3.26416200 & 3.03812500 & -3.38202700 \\
\hline $\mathrm{H}$ & -2.27170500 & 1.76960300 & -2.64224000 \\
\hline $\mathrm{H}$ & -3.26447300 & 3.03809800 & 3.38201400 \\
\hline $\mathrm{H}$ & -4.02642800 & 1.62073800 & 2.63909700 \\
\hline $\mathrm{H}$ & -2.27202300 & 1.76952100 & 2.64231200 \\
\hline $\mathrm{H}$ & -3.08697000 & 7.13669100 & -0.88537000 \\
\hline $\mathrm{H}$ & -4.60886600 & 6.99469200 & -0.00213700 \\
\hline $\mathrm{H}$ & -3.09054800 & 7.13637900 & 0.88734000 \\
\hline $\mathrm{H}$ & -14.11589200 & -1.50782300 & -0.00002200 \\
\hline $\mathrm{H}$ & -12.81213600 & -2.30510700 & -0.88803800 \\
\hline $\mathrm{H}$ & -12.81211000 & -2.30514600 & 0.88792400 \\
\hline $\mathrm{H}$ & -13.76700700 & 0.58223900 & 1.27491700 \\
\hline $\mathrm{H}$ & -12.20142300 & 1.40769300 & 1.30190400 \\
\hline $\mathrm{H}$ & -12.40610300 & -0.11848800 & 2.17370700 \\
\hline $\mathrm{H}$ & -12.40610800 & -0.11844800 & -2.17374500 \\
\hline $\mathrm{H}$ & -13.76699100 & 0.58229700 & -1.27493600 \\
\hline
\end{tabular}




$\begin{array}{lrrr}\mathrm{H} & -12.20138500 & 1.40771200 & -1.30191500 \\ \mathrm{H} & 14.11589200 & 1.50781500 & -0.00012200 \\ \mathrm{H} & 12.81212800 & 2.30503900 & -0.88818000 \\ \mathrm{H} & 12.81212100 & 2.30520200 & 0.88778100 \\ \mathrm{H} & 13.76703000 & -0.58215900 & 1.27495200 \\ \mathrm{H} & 12.20144800 & -1.40761400 & 1.30201900 \\ \mathrm{H} & 12.40614000 & 0.11862400 & 2.17372000 \\ \mathrm{H} & 12.40606900 & 0.11830100 & -2.17373200 \\ \mathrm{H} & 13.76696500 & -0.58239100 & -1.27490100 \\ \mathrm{H} & 12.20135500 & -1.40780200 & -1.30180000\end{array}$


1a open-shell triplet state:

$\mathrm{E}=-2702.544254$ a.u.

$\mathrm{C}$

$\begin{array}{lll}1.24730300 & 2.23999300 & -0.00001100\end{array}$

$\begin{array}{llll}\text { C } & 2.22790800 & 1.24132200 & -0.00001400\end{array}$

C $\quad 1.88427400 \quad-0.13033200 \quad-0.00001200$

C $\quad 0.50119100 \quad-0.52192800 \quad-0.00000300$

$\begin{array}{llll}\text { C } & -0.50119100 & 0.52192800 & 0.00000000\end{array}$

C $\quad-0.08750400 \quad 1.88479100 \quad-0.00000500$

C $\quad 0.08750300 \quad-1.88479100 \quad 0.00000400$

C $\quad-1.24730300 \quad-2.23999300 \quad 0.00000600$

C $\quad-2.22790800 \quad-1.24132200 \quad 0.00000600$

C $\quad-1.88427400 \quad 0.13033200 \quad 0.00000600$

C $\quad-3.68037800 \quad-1.34862900 \quad 0.00000700$

C $\quad 3.68037800 \quad 1.34862900 \quad-0.00001900$

C $\quad 4.49042800 \quad 2.45939500 \quad-0.00001200$

C $\quad 5.89854300 \quad 2.31023100 \quad-0.00001000$

C $\quad 6.46844300 \quad 0.99632300 \quad-0.00002300$

C $\quad 5.63282800 \quad-0.17379200 \quad-0.00003500$

C $\quad 4.21688200 \quad 0.01339400 \quad-0.00002600$

C $\quad 6.76407500 \quad 3.44458600 \quad 0.00000600$

C $\quad 8.12497300 \quad 3.30751200 \quad 0.00001100$

C $\quad 8.73300900 \quad 2.01231800 \quad-0.00000200$

C $\quad 7.88904500 \quad 0.86197400 \quad-0.00002100$

C $\quad 10.13233600 \quad 1.84812600 \quad 0.00000500$

C $\quad 10.73724800 \quad 0.58792400 \quad-0.00000800$

C $\quad 9.89560900 \quad-0.53531000 \quad-0.00003100$

C $\quad 8.49638900 \quad-0.42835400 \quad-0.00003700$

C $\quad 7.63948100 \quad-1.57422900 \quad-0.00005900$

C $\quad 6.27759400 \quad-1.45307500 \quad-0.00005800$

C $\quad-4.49042800 \quad-2.45939500 \quad 0.00000400$ 


\begin{tabular}{|c|c|c|c|}
\hline $\mathrm{C}$ & -5.89854400 & -2.31023100 & 0.00000300 \\
\hline $\mathrm{C}$ & -6.46844300 & -0.99632300 & 0.00000700 \\
\hline $\mathrm{C}$ & -5.63282800 & 0.17379200 & 0.00001300 \\
\hline $\mathrm{C}$ & -4.21688200 & -0.01339400 & 0.00001100 \\
\hline $\mathrm{C}$ & -6.76407600 & -3.44458600 & -0.00000300 \\
\hline $\mathrm{C}$ & -8.12497400 & -3.30751200 & -0.00000400 \\
\hline $\mathrm{C}$ & -8.73300900 & -2.01231800 & 0.00000000 \\
\hline $\mathrm{C}$ & -7.88904500 & -0.86197400 & 0.00000600 \\
\hline $\mathrm{C}$ & -10.13233700 & -1.84812600 & -0.00000100 \\
\hline $\mathrm{C}$ & -10.73724800 & -0.58792400 & 0.00000300 \\
\hline $\mathrm{C}$ & -9.89560900 & 0.53531000 & 0.00000900 \\
\hline $\mathrm{C}$ & -8.49638900 & 0.42835400 & 0.00001100 \\
\hline $\mathrm{C}$ & -7.63948100 & 1.57422900 & 0.00001800 \\
\hline $\mathrm{C}$ & -6.27759400 & 1.45307500 & 0.00001900 \\
\hline $\mathrm{C}$ & 3.11666200 & -0.90417800 & -0.00001900 \\
\hline $\mathrm{C}$ & -3.11666200 & 0.90417800 & 0.00001100 \\
\hline $\mathrm{C}$ & -3.22367900 & 2.38824800 & 0.00001800 \\
\hline $\mathrm{C}$ & 3.22367900 & -2.38824800 & -0.00000400 \\
\hline $\mathrm{C}$ & 3.27277400 & -3.09422900 & -1.22224000 \\
\hline $\mathrm{C}$ & 3.37020500 & -4.48905900 & -1.19842200 \\
\hline $\mathrm{C}$ & 3.42345700 & -5.20706500 & 0.00001700 \\
\hline $\mathrm{C}$ & 3.37031600 & -4.48903900 & 1.19842700 \\
\hline $\mathrm{C}$ & 3.27288200 & -3.09419000 & 1.22222600 \\
\hline $\mathrm{C}$ & -3.27280800 & 3.09420500 & 1.22225800 \\
\hline $\mathrm{C}$ & -3.37023900 & 4.48904200 & 1.19845900 \\
\hline $\mathrm{C}$ & -3.42345600 & 5.20706600 & 0.00003600 \\
\hline $\mathrm{C}$ & -3.37028000 & 4.48905600 & -1.19839000 \\
\hline $\mathrm{C}$ & -3.27284800 & 3.09421400 & -1.22220800 \\
\hline $\mathrm{C}$ & -3.21478700 & 2.35987000 & -2.54228000 \\
\hline $\mathrm{C}$ & -3.21470300 & 2.35984300 & 2.54231900 \\
\hline
\end{tabular}




\begin{tabular}{|c|c|c|c|}
\hline $\mathrm{C}$ & -3.55920900 & 6.71214200 & 0.00005700 \\
\hline $\mathrm{C}$ & 3.21463100 & -2.35988200 & -2.54230700 \\
\hline $\mathrm{C}$ & 3.21485900 & -2.35983100 & 2.54229200 \\
\hline $\mathrm{C}$ & 3.55921000 & -6.71214200 & -0.00000700 \\
\hline $\mathrm{C}$ & 12.26558700 & 0.39625100 & -0.00000100 \\
\hline $\mathrm{C}$ & 13.02535500 & 1.73647100 & 0.00004600 \\
\hline $\mathrm{C}$ & 12.68472700 & -0.39416600 & 1.26235400 \\
\hline $\mathrm{C}$ & 12.68474200 & -0.39408800 & -1.26240000 \\
\hline $\mathrm{C}$ & -12.26558700 & -0.39625100 & -0.00000200 \\
\hline $\mathrm{C}$ & -13.02535500 & -1.73647100 & -0.00000900 \\
\hline $\mathrm{C}$ & -12.68474200 & 0.39412300 & 1.26237500 \\
\hline $\mathrm{C}$ & -12.68472800 & 0.39413000 & -1.26237900 \\
\hline $\mathrm{H}$ & 1.52765900 & 3.29044300 & -0.00001400 \\
\hline $\mathrm{H}$ & -0.83854700 & 2.66272800 & -0.00000500 \\
\hline $\mathrm{H}$ & 0.83854600 & -2.66272800 & 0.00000800 \\
\hline $\mathrm{H}$ & -1.52765900 & -3.29044300 & 0.00001000 \\
\hline $\mathrm{H}$ & 4.06703300 & 3.46151800 & -0.00000400 \\
\hline $\mathrm{H}$ & 6.31420300 & 4.43472300 & 0.00001500 \\
\hline $\mathrm{H}$ & 8.76654200 & 4.18541400 & 0.00002500 \\
\hline $\mathrm{H}$ & 10.74041500 & 2.74669400 & 0.00002300 \\
\hline $\mathrm{H}$ & 10.32609400 & -1.53348900 & -0.00004500 \\
\hline $\mathrm{H}$ & 8.09407500 & -2.56230000 & -0.00007600 \\
\hline $\mathrm{H}$ & 5.66820600 & -2.34739800 & -0.00007400 \\
\hline $\mathrm{H}$ & -4.06703400 & -3.46151800 & 0.00000000 \\
\hline $\mathrm{H}$ & -6.31420300 & -4.43472300 & -0.00000600 \\
\hline $\mathrm{H}$ & -8.76654200 & -4.18541400 & -0.00000900 \\
\hline $\mathrm{H}$ & -10.74041500 & -2.74669400 & -0.00000500 \\
\hline $\mathrm{H}$ & -10.32609400 & 1.53348900 & 0.00001100 \\
\hline $\mathrm{H}$ & -8.09407600 & 2.56230000 & 0.00002200 \\
\hline $\mathrm{H}$ & -5.66820600 & 2.34739800 & 0.00002400 \\
\hline
\end{tabular}




\begin{tabular}{|c|c|c|c|}
\hline $\mathrm{H}$ & 3.40350700 & -5.02847100 & -2.14333000 \\
\hline $\mathrm{H}$ & 3.40371100 & -5.02843000 & 2.14334000 \\
\hline $\mathrm{H}$ & -3.40357100 & 5.02843800 & 2.14337300 \\
\hline $\mathrm{H}$ & -3.40364500 & 5.02846300 & -2.14329700 \\
\hline $\mathrm{H}$ & -3.26426100 & 3.05996000 & -3.38202900 \\
\hline $\mathrm{H}$ & -2.28882200 & 1.78055300 & -2.63858600 \\
\hline $\mathrm{H}$ & -4.04444900 & 1.65032400 & -2.64328800 \\
\hline $\mathrm{H}$ & -2.28872600 & 1.78054000 & 2.63859200 \\
\hline $\mathrm{H}$ & -3.26416700 & 3.05991900 & 3.38207900 \\
\hline $\mathrm{H}$ & -4.04435000 & 1.65028100 & 2.64333700 \\
\hline $\mathrm{H}$ & -3.09343200 & 7.15679400 & 0.88609700 \\
\hline $\mathrm{H}$ & -3.09450600 & 7.15671500 & -0.88658300 \\
\hline $\mathrm{H}$ & -4.61436100 & 7.01762300 & 0.00069300 \\
\hline $\mathrm{H}$ & 4.04426900 & -1.65031300 & -2.64335200 \\
\hline $\mathrm{H}$ & 3.26408200 & -3.05996800 & -3.38206100 \\
\hline $\mathrm{H}$ & 2.28864600 & -1.78059000 & -2.63856500 \\
\hline $\mathrm{H}$ & 3.26434600 & -3.05991100 & 3.38204700 \\
\hline $\mathrm{H}$ & 4.04453000 & -1.65029100 & 2.64327300 \\
\hline $\mathrm{H}$ & 2.28890200 & -1.78050400 & 2.63861300 \\
\hline $\mathrm{H}$ & 3.09211300 & -7.15691800 & -0.88529500 \\
\hline $\mathrm{H}$ & 4.61436000 & -7.01762200 & -0.00220100 \\
\hline $\mathrm{H}$ & 3.09582900 & -7.15659100 & 0.88738100 \\
\hline $\mathrm{H}$ & 14.10499900 & 1.54846600 & 0.00005700 \\
\hline $\mathrm{H}$ & 12.79566100 & 2.33634800 & -0.88796100 \\
\hline $\mathrm{H}$ & 12.79563300 & 2.33630100 & 0.88807700 \\
\hline $\mathrm{H}$ & 13.77118600 & -0.54466000 & 1.27469700 \\
\hline $\mathrm{H}$ & 12.21142600 & -1.38083300 & 1.30211300 \\
\hline $\mathrm{H}$ & 12.40583200 & 0.14680300 & 2.17377800 \\
\hline $\mathrm{H}$ & 12.40587000 & 0.14694500 & -2.17379300 \\
\hline $\mathrm{H}$ & 13.77120000 & -0.54459500 & -1.27473300 \\
\hline
\end{tabular}




$\begin{array}{lrrr}\mathrm{H} & 12.21142800 & -1.38074600 & -1.30223100 \\ \mathrm{H} & -14.10499900 & -1.54846600 & -0.00000400 \\ \mathrm{H} & -12.79565100 & -2.33631800 & -0.88803400 \\ \mathrm{H} & -12.79564300 & -2.33633100 & 0.88800500 \\ \mathrm{H} & -13.77120100 & 0.54462000 & 1.27470900 \\ \mathrm{H} & -12.21143900 & 1.38078700 & 1.30217400 \\ \mathrm{H} & -12.40586000 & -0.14687800 & 2.17378300 \\ \mathrm{H} & -12.40584200 & -0.14687000 & -2.17378700 \\ \mathrm{H} & -13.77118600 & 0.54463600 & -1.27472100 \\ \mathrm{H} & -12.21141500 & 1.38079100 & -1.30217000\end{array}$




\subsection{Spin density}

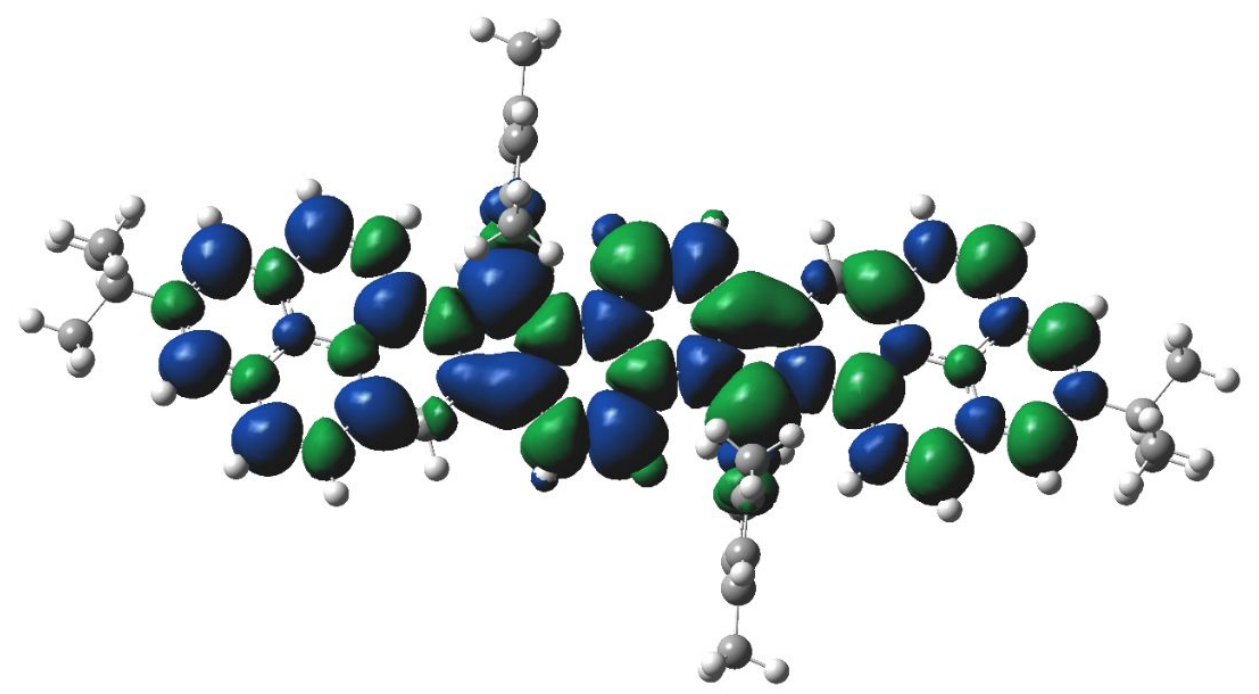

Figure S30. Visualization of the spin density of $\mathbf{1 a .}$

1a Mulliken charges and spin densities:
12
1 C $\quad-0.220803-0.135023$
2 C $\quad 0.081709 \quad 0.181870$
3 C $-0.018676-0.165432$
$\begin{array}{llll}4 & \mathrm{C} & 0.120657 & 0.157339\end{array}$
$\begin{array}{llll}5 & \mathrm{C} & 0.120658 & -0.157339\end{array}$
$\begin{array}{llll}6 & C & -0.232864 & 0.227002\end{array}$
$\begin{array}{llll}7 & C & -0.232864 & -0.227002\end{array}$
$\begin{array}{llll}8 & C & -0.220804 & 0.135023\end{array}$
9 C $0.081709-0.181869$
10 C $\quad-0.018676 \quad 0.165432$
$\begin{array}{llll}11 & C & 0.098904 & -0.048700\end{array}$
$\begin{array}{llll}12 & \mathrm{C} & 0.098903 & 0.048699\end{array}$
$\begin{array}{llll}13 & C & -0.308950 & -0.012465\end{array}$
$\begin{array}{llll}14 & \mathrm{C} & 0.162450 & 0.082017\end{array}$
$\begin{array}{llll}15 & \mathrm{C} & 0.013107 & -0.032473\end{array}$ 


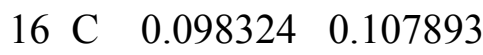

$\begin{array}{llll}17 & C & -0.042833 & -0.102866\end{array}$

$\begin{array}{llll}18 & C & -0.196277 & -0.040656\end{array}$

$\begin{array}{lllll}19 & C & -0.203864 & 0.072721\end{array}$

$20 \mathrm{C} \quad 0.179483 \quad-0.027815$

$\begin{array}{llll}21 & C & -0.039366 & 0.016973\end{array}$

$\begin{array}{llll}22 & \mathrm{C} & -0.314107 & 0.058554\end{array}$

23 C $0.201049-0.029360$

$\begin{array}{llll}24 & \mathrm{C} & -0.302267 & 0.069371\end{array}$

25 C $0.185119 \quad-0.034456$

$\begin{array}{llll}26 & \mathrm{C} & -0.216358 & 0.072639\end{array}$

$\begin{array}{llll}27 & C & -0.193413 & -0.050478\end{array}$

$\begin{array}{llll}28 & \mathrm{C} & -0.308950 & 0.012465\end{array}$

29 C $\quad 0.162450 \quad-0.082017$

$\begin{array}{llll}30 & \mathrm{C} & 0.013106 & 0.032473\end{array}$

31 C $0.098325 \quad-0.107893$

$\begin{array}{llll}32 & \mathrm{C} & -0.042834 & 0.102866\end{array}$

$\begin{array}{llll}33 & \mathrm{C} & -0.196277 & 0.040656\end{array}$

34 C $-0.203864-0.072721$

$\begin{array}{llll}35 & \mathrm{C} & 0.179483 & 0.027815\end{array}$

$\begin{array}{llll}36 & C & -0.039366 & -0.016973\end{array}$

$\begin{array}{llll}37 & C & -0.314107 & -0.058554\end{array}$

$\begin{array}{llll}38 & \mathrm{C} & 0.201048 & 0.029360\end{array}$

$\begin{array}{lllll}39 & C & -0.302267 & -0.069371\end{array}$

$\begin{array}{llll}40 & \mathrm{C} & 0.185119 & 0.034456\end{array}$

$\begin{array}{lllll}41 & C & -0.216357 & -0.072638\end{array}$

$\begin{array}{lllll}42 & C & -0.193413 & 0.050478\end{array}$

$\begin{array}{lllll}43 & \mathrm{C} & -0.069187 & 0.384679\end{array}$

$\begin{array}{lllll}44 & C & -0.069187 & -0.384680\end{array}$

$\begin{array}{llll}45 & C & -0.135724 & 0.032072\end{array}$ 
46 C $\quad-0.135723-0.032072$

$\begin{array}{llll}47 & \mathrm{C} & 0.197092 & 0.011361\end{array}$

$\begin{array}{llll}48 & \mathrm{C} & -0.267723 & -0.001522\end{array}$

$\begin{array}{llll}49 & \mathrm{C} & 0.210938 & 0.001684\end{array}$

$\begin{array}{llll}50 & \mathrm{C} & -0.267765 & -0.001521\end{array}$

$\begin{array}{llll}51 & \mathrm{C} & 0.197108 & 0.011361\end{array}$

52 C $0.197098-0.011361$

$\begin{array}{llll}53 & \mathrm{C} & -0.267738 & 0.001522\end{array}$

$\begin{array}{llll}54 & \mathrm{C} & 0.210938 & -0.001684\end{array}$

$\begin{array}{llll}55 & \mathrm{C} & -0.267750 & 0.001521\end{array}$

56 C $0.197103-0.011361$

$\begin{array}{lllll}57 & \mathrm{C} & -0.535850 & 0.000261\end{array}$

$\begin{array}{llll}58 & \mathrm{C} & -0.535849 & 0.000261\end{array}$

$\begin{array}{llll}59 & \mathrm{C} & -0.532118 & 0.000105\end{array}$

60 C $-0.535848-0.000261$

$\begin{array}{llll}61 & C & -0.535851 & -0.000261\end{array}$

62 C $-0.532118-0.000105$

$\begin{array}{llll}63 & \mathrm{C} & 0.015104 & 0.001351\end{array}$

64 C $-0.455822-0.000022$

$\begin{array}{llll}65 & C & -0.439013 & -0.000323\end{array}$

66 C $-0.439014-0.000323$

67 C $0.015105 \quad-0.001351$

$\begin{array}{llll}68 & \mathrm{C} & -0.455822 & 0.000022\end{array}$

$\begin{array}{llll}69 & C & -0.439011 & 0.000323\end{array}$

$\begin{array}{llll}70 & C & -0.439015 & 0.000323\end{array}$

$\begin{array}{llll}71 & \mathrm{H} & 0.133277 & 0.005329\end{array}$

$\begin{array}{llll}72 & \mathrm{H} & 0.163140 & -0.009936\end{array}$

$\begin{array}{llll}73 & \mathrm{H} & 0.163140 & 0.009936\end{array}$

$\begin{array}{llll}74 & \mathrm{H} & 0.133277 & -0.005329\end{array}$

$\begin{array}{llll}75 & \mathrm{H} & 0.130583 & 0.000253\end{array}$ 
$\begin{array}{llll}76 & \mathrm{H} & 0.127823 & 0.001495\end{array}$

$\begin{array}{llll}77 & \mathrm{H} & 0.128163 & -0.003255\end{array}$

$78 \mathrm{H} \quad 0.124534 \quad-0.002661$

$\begin{array}{llll}79 & \mathrm{H} & 0.123797 & -0.003121\end{array}$

$\begin{array}{llll}80 & \mathrm{H} & 0.127712 & -0.003268\end{array}$

$\begin{array}{llll}81 \mathrm{H} & 0.160075 & 0.001874\end{array}$

$\begin{array}{llll}82 & \mathrm{H} & 0.130583 & -0.000253\end{array}$

$\begin{array}{llll}83 & \mathrm{H} & 0.127823 & -0.001495\end{array}$

$\begin{array}{lll}84 \mathrm{H} & 0.128163 & 0.003255\end{array}$

$\begin{array}{llll}85 & \mathrm{H} & 0.124534 & 0.002661\end{array}$

$\begin{array}{llll}86 \mathrm{H} & 0.123797 & 0.003121\end{array}$

$\begin{array}{llll}87 & \mathrm{H} & 0.127712 & 0.003268\end{array}$

88 H $0.160075 \quad-0.001874$

$\begin{array}{llll}89 & \mathrm{H} & 0.115215 & 0.000763\end{array}$

$90 \mathrm{H} \quad 0.1152160 .000763$

$91 \mathrm{H} \quad 0.115215-0.000763$

$92 \mathrm{H} \quad 0.115216-0.000763$

$\begin{array}{llll}93 & \mathrm{H} & 0.150162 & -0.000371\end{array}$

$94 \mathrm{H} \quad 0.171143-0.000132$

$\begin{array}{llll}95 & \mathrm{H} & 0.171505 & 0.000029\end{array}$

$\begin{array}{llll}96 & \mathrm{H} & 0.171144 & -0.000132\end{array}$

$\begin{array}{llll}97 & \mathrm{H} & 0.150162 & -0.000371\end{array}$

$\begin{array}{llll}98 & \mathrm{H} & 0.171504 & 0.000029\end{array}$

$\begin{array}{llll}99 & \mathrm{H} & 0.156627 & -0.000017\end{array}$

$100 \mathrm{H} \quad 0.156620-0.000017$

$\begin{array}{lllll}101 & \mathrm{H} & 0.164965 & -0.000084\end{array}$

$102 \mathrm{H} \quad 0.171504-0.000029$

$\begin{array}{lllll}103 & \mathrm{H} & 0.150161 & 0.000371\end{array}$

$\begin{array}{llll}104 & \mathrm{H} & 0.171144 & 0.000132\end{array}$

$\begin{array}{llll}105 & \mathrm{H} & 0.150163 & 0.000371\end{array}$ 
106 H $0.171505-0.000029$

$107 \mathrm{H} \quad 0.171143 \quad 0.000132$

$\begin{array}{llll}108 \mathrm{H} & 0.156636 & 0.000017\end{array}$

$109 \mathrm{H} \quad 0.164965 \quad 0.000084$

$\begin{array}{llll}110 \mathrm{H} & 0.156610 & 0.000017\end{array}$

$\begin{array}{llll}111 \mathrm{H} & 0.141310 & 0.000001\end{array}$

$\begin{array}{llll}112 & \mathrm{H} & 0.145897 & 0.000003\end{array}$

$113 \mathrm{H} \quad 0.145897 \quad 0.000003$

$114 \mathrm{H} \quad 0.138307 \quad 0.000006$

$\begin{array}{llll}115 & \mathrm{H} & 0.143621 & 0.000053\end{array}$

$\begin{array}{llll}116 & \mathrm{H} & 0.148235 & -0.000027\end{array}$

117 H $0.148235-0.000027$

$\begin{array}{llll}118 \mathrm{H} & 0.138307 & 0.000006\end{array}$

$\begin{array}{llll}119 & \mathrm{H} & 0.143621 & 0.000053\end{array}$

$120 \mathrm{H} \quad 0.141310-0.000001$

$121 \mathrm{H} \quad 0.145897 \quad-0.000003$

$\begin{array}{llll}122 & \mathrm{H} & 0.145897 & -0.000003\end{array}$

$123 \mathrm{H} \quad 0.138307-0.000006$

$124 \mathrm{H} \quad 0.143621-0.000053$

$\begin{array}{llll}125 & \mathrm{H} & 0.148235 & 0.000027\end{array}$

$\begin{array}{llll}126 \mathrm{H} & 0.148236 & 0.000027\end{array}$

$127 \mathrm{H} \quad 0.138307 \quad-0.000006$

$128 \mathrm{H} \quad 0.143621-0.000053$

Sum of Mulliken charges $=0.00000 \quad 0.00000$ 


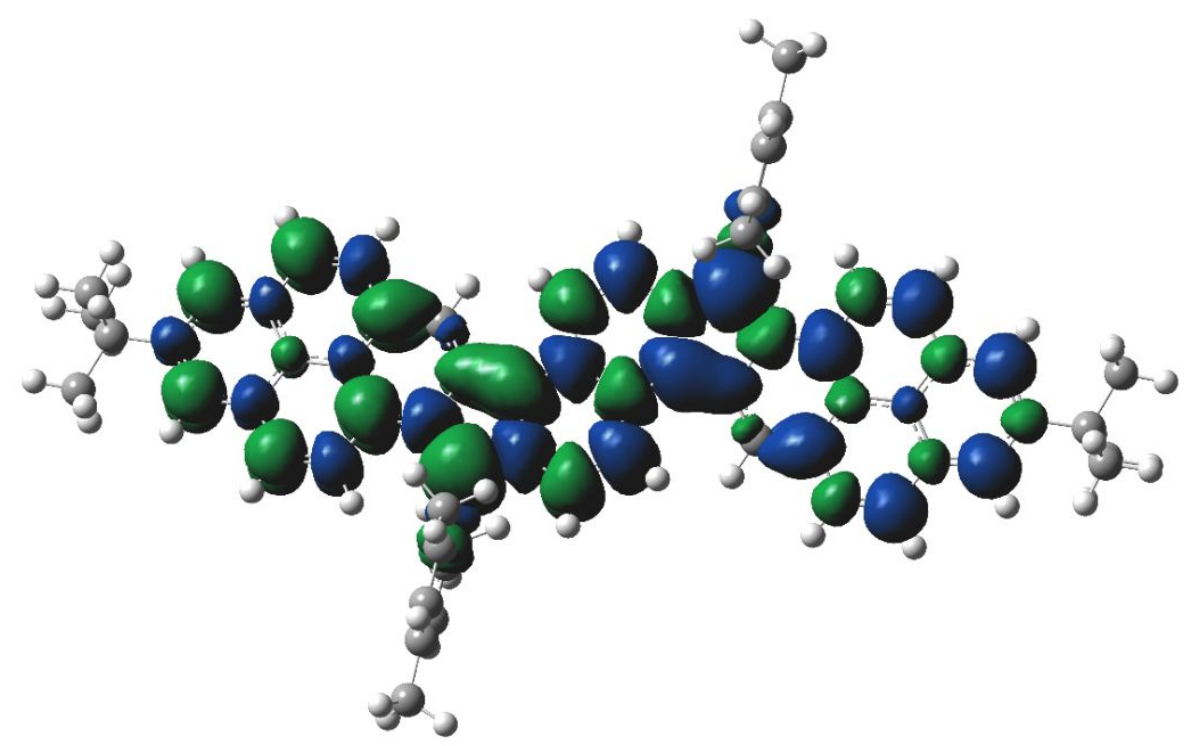

Figure S31. Visualization of the spin density of $\mathbf{1 b}$.

1b Mulliken charges and spin densities:
$1 \quad 2$
1 C $\quad-0.201877-0.083760$
2 C $0.104454 \quad 0.124700$
$\begin{array}{llll}3 & C & -0.097912 & -0.156228\end{array}$
4 C $\quad 0.110550 \quad 0.101992$
5 C $\quad 0.110550-0.101992$
$\begin{array}{llll}6 & C & -0.188104 & 0.082834\end{array}$
7 C $\quad-0.188105-0.082834$
8 C $\quad-0.201876 \quad 0.083760$
9 C $0.104454-0.124700$
$\begin{array}{llll}10 & \mathrm{C} & -0.097913 & 0.156227\end{array}$
$\begin{array}{llll}11 & \mathrm{C} & 0.115693 & 0.040323\end{array}$
12 C $0.115694-0.040323$
$\begin{array}{llll}13 & \mathrm{C} & -0.056512 & 0.082026\end{array}$
14 C $0.106409 \quad-0.081763$
$\begin{array}{llll}15 & \mathrm{C} & 0.006143 & 0.024974\end{array}$
16 C $0.168180 \quad-0.064627$ 
$\begin{array}{llll}17 & C & -0.320689 & 0.007550\end{array}$

$\begin{array}{llll}18 & C & -0.193199 & 0.038525\end{array}$

$\begin{array}{llll}19 & C & -0.215427 & -0.054260\end{array}$

$\begin{array}{llll}20 & \mathrm{C} & 0.189582 & 0.026174\end{array}$

$\begin{array}{llll}21 & C & -0.036575 & -0.012883\end{array}$

$\begin{array}{lllll}22 & C & -0.315495 & -0.049789\end{array}$

$\begin{array}{llll}23 & \mathrm{C} & 0.201744 & 0.022539\end{array}$

$\begin{array}{llll}24 & C & -0.302081 & -0.047843\end{array}$

$\begin{array}{llll}25 & \mathrm{C} & 0.176900 & 0.021582\end{array}$

$\begin{array}{llll}26 & C & -0.202299 & -0.057474\end{array}$

$\begin{array}{llll}27 & \mathrm{C} & -0.198388 & 0.031987\end{array}$

$\begin{array}{llll}28 & C & -0.056511 & -0.082026\end{array}$

$\begin{array}{llll}29 & \mathrm{C} & 0.106409 & 0.081763\end{array}$

$\begin{array}{llll}30 & \mathrm{C} & 0.006143 & -0.024974\end{array}$

$\begin{array}{llll}31 & \mathrm{C} & 0.168180 & 0.064627\end{array}$

32 C $-0.320689-0.007551$

$\begin{array}{llll}33 & C & -0.193199 & -0.038525\end{array}$

$\begin{array}{llll}34 & C & -0.215427 & 0.054260\end{array}$

$\begin{array}{llll}35 & \mathrm{C} & 0.189583 & -0.026174\end{array}$

$\begin{array}{llll}36 & \mathrm{C} & -0.036576 & 0.012884\end{array}$

$\begin{array}{llll}37 & C & -0.315496 & 0.049789\end{array}$

$\begin{array}{llll}38 & \mathrm{C} & 0.201744 & -0.022539\end{array}$

$\begin{array}{lllll}39 & \mathrm{C} & -0.302081 & 0.047843\end{array}$

40 C $0.176900 \quad-0.021582$

$\begin{array}{llll}41 & C & -0.202300 & 0.057475\end{array}$

$\begin{array}{lllll}42 & C & -0.198388 & -0.031987\end{array}$

$\begin{array}{lllll}43 & C & -0.082226 & 0.292559\end{array}$

$\begin{array}{llll}44 & C & -0.082225 & -0.292559\end{array}$

$\begin{array}{llll}45 & C & -0.103174 & 0.024539\end{array}$

$\begin{array}{llll}46 & C & -0.103174 & -0.024539\end{array}$ 


$$
\begin{array}{llll}
47 & \mathrm{C} & 0.190786 & -0.008483 \\
48 & \mathrm{C} & -0.269000 & 0.000889 \\
49 & \mathrm{C} & 0.214045 & -0.001146 \\
50 & \mathrm{C} & -0.270240 & 0.000874 \\
51 & \mathrm{C} & 0.192584 & -0.008482 \\
52 & \mathrm{C} & 0.192586 & 0.008482 \\
53 & \mathrm{C} & -0.270245 & -0.000874 \\
54 & \mathrm{C} & 0.214045 & 0.001146 \\
55 & \mathrm{C} & -0.268994 & -0.000889 \\
56 & \mathrm{C} & 0.190783 & 0.008483 \\
57 & \mathrm{C} & -0.534620 & 0.000141 \\
58 & \mathrm{C} & -0.534720 & 0.000152 \\
59 & \mathrm{C} & -0.532371 & 0.000071 \\
60 & \mathrm{C} & -0.534719 & -0.000151 \\
61 & \mathrm{C} & -0.534620 & -0.000141 \\
62 & \mathrm{C} & -0.532372 & -0.000071 \\
63 & \mathrm{C} & 0.014504 & -0.001216 \\
64 & \mathrm{C} & -0.455833 & -0.000042 \\
65 & \mathrm{C} & -0.438908 & 0.000238 \\
66 & \mathrm{C} & -0.438828 & 0.000238 \\
67 & \mathrm{C} & 0.014504 & 0.001216 \\
68 & \mathrm{C} & -0.455833 & 0.000042 \\
69 & \mathrm{C} & -0.438909 & -0.000238 \\
70 & \mathrm{C} & -0.438827 & -0.000238 \\
71 & \mathrm{H} & 0.141285 & 0.003354 \\
72 & \mathrm{H} & 0.135998 & -0.003600 \\
73 & \mathrm{H} & 0.135997 & 0.003600 \\
74 & \mathrm{H} & 0.141285 & -0.003354 \\
76 \mathrm{H} & 0.142701 & -0.000187 \\
\hline & 0.159778 & -0.001431
\end{array}
$$


$\begin{array}{llll}77 \mathrm{H} & 0.129253 & 0.002436\end{array}$

$\begin{array}{llll}78 & \mathrm{H} & 0.125961 & 0.002202\end{array}$

$\begin{array}{llll}79 \mathrm{H} & 0.123646 & 0.002210\end{array}$

$\begin{array}{llll}80 & \mathrm{H} & 0.128614 & 0.002569\end{array}$

$\begin{array}{llll}81 & \mathrm{H} & 0.128555 & -0.001172\end{array}$

$\begin{array}{llll}82 & \mathrm{H} & 0.142701 & 0.000187\end{array}$

$\begin{array}{llll}83 & \mathrm{H} & 0.159777 & 0.001431\end{array}$

$\begin{array}{llll}84 & \mathrm{H} & 0.129253 & -0.002436\end{array}$

$\begin{array}{llll}85 & \mathrm{H} & 0.125961 & -0.002202\end{array}$

$86 \mathrm{H} \quad 0.123646-0.002210$

$\begin{array}{llll}87 \mathrm{H} & 0.128614 & -0.002569\end{array}$

$\begin{array}{llll}88 & \mathrm{H} & 0.128555 & 0.001172\end{array}$

$89 \mathrm{H} \quad 0.114293 \quad-0.000570$

$\begin{array}{llll}90 & \mathrm{H} & 0.114219 & -0.000573\end{array}$

$\begin{array}{llll}91 & \mathrm{H} & 0.114220 & 0.000573\end{array}$

$\begin{array}{llll}92 & \mathrm{H} & 0.114293 & 0.000570\end{array}$

$\begin{array}{llll}93 & \mathrm{H} & 0.150349 & -0.000261\end{array}$

$94 \mathrm{H} \quad 0.1712650 .000006$

$\begin{array}{llll}95 & \mathrm{H} & 0.168614 & 0.000038\end{array}$

$\begin{array}{llll}96 & \mathrm{H} & 0.170462 & -0.000028\end{array}$

$\begin{array}{llll}97 & \mathrm{H} & 0.150481 & -0.000264\end{array}$

$\begin{array}{llll}98 & \mathrm{H} & 0.169171 & 0.000069\end{array}$

$\begin{array}{llll}99 & \mathrm{H} & 0.156633 & -0.000016\end{array}$

$100 \mathrm{H} \quad 0.155968 \quad-0.000007$

$101 \mathrm{H} \quad 0.164881-0.000059$

$\begin{array}{llll}102 \mathrm{H} & 0.150481 & 0.000264\end{array}$

$103 \mathrm{H} \quad 0.169171-0.000069$

$\begin{array}{lll}104 \mathrm{H} & 0.170461 & 0.000028\end{array}$

$105 \mathrm{H} \quad 0.168614-0.000038$

$\begin{array}{lll}106 \mathrm{H} & 0.150349 & 0.000261\end{array}$ 
$107 \mathrm{H} \quad 0.171265-0.000006$

$\begin{array}{llll}108 \mathrm{H} & 0.155965 & 0.000007\end{array}$

$109 \mathrm{H} \quad 0.164880 \quad 0.000059$

$\begin{array}{llll}110 \mathrm{H} & 0.156637 & 0.000016\end{array}$

$111 \mathrm{H} \quad 0.141594 \quad 0.000004$

$112 \mathrm{H} \quad 0.146155 \quad-0.000017$

$113 \mathrm{H} \quad 0.146220 \quad-0.000017$

$114 \mathrm{H} \quad 0.1385820 .000142$

$115 \mathrm{H} \quad 0.143433-0.000024$

$116 \mathrm{H} \quad 0.148403 \quad 0.000010$

$\begin{array}{lll}117 \mathrm{H} & 0.148403 & 0.000010\end{array}$

$118 \mathrm{H} \quad 0.138593 \quad 0.000142$

119 H $0.143393 \quad-0.000024$

$120 \mathrm{H} \quad 0.141594 \quad-0.000004$

$\begin{array}{llll}121 & \mathrm{H} & 0.146155 & 0.000017\end{array}$

$\begin{array}{llll}122 & \mathrm{H} & 0.146220 & 0.000017\end{array}$

$123 \mathrm{H} \quad 0.138581 \quad-0.000142$

$124 \mathrm{H} \quad 0.143433 \quad 0.000024$

$125 \mathrm{H} \quad 0.148404-0.000010$

$126 \mathrm{H} \quad 0.148403-0.000010$

127 H $0.138593-0.000142$

$\begin{array}{llll}128 \mathrm{H} & 0.143393 & 0.000024\end{array}$

Sum of Mulliken charges $=0.00000 \quad 0.00000$ 


\section{7) References}

[1] Gaussian 09, Revision A.02, M. J. Frisch, G. W. Trucks, H. B. Schlegel, G. E. Scuseria, M. A. Robb, J. R. Cheeseman, G. Scalmani, V. Barone, G. A. Petersson, H. Nakatsuji, X. Li, M. Caricato, A. Marenich, J. Bloino, B. G. Janesko, R. Gomperts, B. Mennucci, H. P. Hratchian, J. V. Ortiz, A. F. Izmaylov, J. L. Sonnenberg, D. Williams-Young, F. Ding, F. Lipparini, F. Egidi, J. Goings, B. Peng, A. Petrone, T. Henderson, D. Ranasinghe, V. G. Zakrzewski, J. Gao, N. Rega, G. Zheng, W. Liang, M. Hada, M. Ehara, K. Toyota, R. Fukuda, J. Hasegawa, M. Ishida, T. Nakajima, Y. Honda, O. Kitao, H. Nakai, T. Vreven, K. Throssell, J. A. Montgomery, Jr., J. E. Peralta, F. Ogliaro, M. Bearpark, J. J. Heyd, E. Brothers, K. N. Kudin, V. N. Staroverov, T. Keith, R. Kobayashi, J. Normand, K. Raghavachari, A. Rendell, J. C. Burant, S. S. Iyengar, J. Tomasi, M. Cossi, J. M. Millam, M. Klene, C. Adamo, R. Cammi, J. W. Ochterski, R. L. Martin, K. Morokuma, O. Farkas, J. B. Foresman, and D. J. Fox, Gaussian, Inc., Wallingford CT, 2016.

[2] (a) Yamaguchi, K.; Jensen, F.; Dorigo, A.; Houk, K. N., A spin correction procedure forunrestricted Hartree-Fock and Møller-Plesset wavefunctions for singlet diradicals and polyradicals. Chem. Phys. Lett. 1988, 49, 537-542. (b) Yamaguchi, K.; Takahara, Y.; S66 Fueno, T.; Houk, K. N., Extended Hartree-Fock (EHF) theory of chemical reactions. Theor. Chim. Acta 1988, 73, 337-364.

[3] D. Geuenich, K. Hess, F. Köhler, R. Herges, Anisotropy of the Induced Current Density (ACID), a General Method To Quantify and Visualize Electronic Delocalization. Chem. Rev. 2005, 105, 3758-3772.

[4] (a) Z. Chen, C. S. Wannere, C. Corminboeuf, R. Puchta, P. v. R.Schleyer, NucleusIndependent Chemical Shifts (NICS) as an Aromaticity Criterion. Chem. Rev. 2015, 105, 3842-3888. (b) H. Fallah-Bagher-Shaidaei, S. S. Wannere, C. Corminboeuf, R., Puchta, P. v. R. Schleyer, Which NICS Aromaticity Index for Planar $\pi$ Rings Is Best? Org. Lett. 2006, 8, 863-866. 